\title{
DIVERSITY AND ECOLOGICAL ANALYSIS OF SERPENTINE FLORA IN THE KOSOVO SECTION OF THE IBAR RIVER VALLEY - COMPARISION WITH THE FLORA OF NEARBY REGIONS
}

\author{
ProdAnOviĆ, D. ${ }^{1 *}$ - KRIVOŠEJ, Z. ${ }^{2}$ - AMIDŽIĆ, L. $^{3}$ - ĆIRIĆ, S. ${ }^{1}$ - BIBERDŽIĆ, M. ${ }^{1}-$ KRSTIĆ, Z. $^{2}$ \\ ${ }^{1}$ University of Priština, Faculty of Agriculture Lešak, Kopaonička Street bb, 38219 Lešak, \\ Serbia \\ ${ }^{2}$ University of Priština, Faculty of Natural Science Lole Ribara Street, No. 29, 38220 Kosovska \\ Mitrovica, Serbia \\ ${ }^{3}$ Singidunum University, Environment and sustainable development studies, Danijelova Street, \\ No. 32, 11000 Belgrade, Serbia \\ *Corresponding author \\ e-mail: danijela.prodanovic@gmail.com \\ (Received $7^{\text {th }}$ May 2020; accepted $20^{\text {th }}$ Aug 2020)
}

\begin{abstract}
Formation of serpentine flora and vegetation is primarily influenced by local geology and they represent an extraordinary area for botanical research studies (from both taxonomical and ecological perspective). During a sixteen-year research of serpentine terrain flora of the Ibar river middle-stream valley in northern Kosovo and Metohija, the existence of 882 taxa grouped into 83 families and 386 genera has been proven. The collected serpentine flora was analysed on taxonomical, ecological and phytogeographical basis. Out of the total number of identified taxa, $73(8.27 \%)$ are endemic, sub-endemic, relict and endomorelic. 31 taxa belong to a group of internationally significant vascular plants. Of these, 14 taxa have been protected by CITES Convention. The taxonomic structure of the serpentine flora of the Ibar river middle-stream valley is compared to the serpentine flora of Mt. Studena and Goleš Mt. (parts of Ibar serpentine massive).
\end{abstract}

Keywords: floristic composition, Ibar serpentine massif, endemism, floristic similarity, Serbia

\section{Introduction}

Serpentine is technically a mineral, but the same word is often used for all ultramafic rocks, the soils that form from them, and the unique ecosystems that form on them (Harrison and Rajakaruna, 2011). Serpentine is a ferromagnesian silicate mineral which contains high concentrations of nickel $(\mathrm{Ni})$ and often chromium $(\mathrm{Cr})$ and cobalt $(\mathrm{Co})$ in a form available for plants (Westerbergh and Saura, 1992). Serpentine soils are often deficient in plant essential nutrients such as nitrogen $(\mathrm{N})$, phosphorus $(\mathrm{P})$, potassium (K), and sulfur (S) (Rajakaruna and Boyd, 2014), while ratio of calcium (Ca): magnesium $(\mathrm{Mg})$ is less than 1.0, and $\mathrm{pH}$ values range from basic to ultrabasic (Brooks, 1987; Selvi, 2007; Pavlova, 2007; Bani et al., 2013). Owing to this chemical composition, Novák (1926) described serpentine ranges as "dead rocks". Serpentine soils are typically recognized on the landscape as patchily distributed rock outcrops with stunted vegetation (Anacker, 2014). Serpentine outcrops are often steep and comparatively rocky, making them particularly vulnerable to erosion, which results in shallow soils (Brady et al., 2005) and stony structure (Kurt et al., 2013).

The serpentinite is rather unfavorable to plant growth, and its physical conditions also are inhospitable for many plants (Bani et al., 2013). However, plants would not be 
what they are, if they weren't able to conquer any tolerably suitable substratum by adapting to it (Vasić and Diklić, 2001). Plants adapted to grow under these constraints are called serpentinophytes (Selvi, 2007), distinguished by characteristic structuralmorphological adaptations (Stevanović and Jakovljević, 2014); they posses capacity for removal and accumulation of metals (Branković et al., 2017). Many serpentine species are xeromorphic, and water deficiency has been suggested as another stress factor of many serpentine soils (Tumi, 2013). The serpentine flora contains both basophilous and acidophilous plants (Marin and Tatić, 2001). Vegetation growing on serpentinised rocks is often reduced in height, biomass, and ground cover (Gavrilović et al., 2017).

Ultramafic outcrops (also called "serpentine"), formed during tectonic movements, are widespread but sparse, covering roughly 3\% of the Earth's surface (Guillot and Hattori, 2013). The largest serpentine areas in Europe are in the Balkans (Stevanović et al., 2003), with an estimated size of over $1,300 \mathrm{~km}^{2}$ (Pustahija, 2011).

The serpentine areas on the Balkan Peninsula traverse discontinuously from the northwest to central Bosnia, over western and central Serbia, Metohija, Albania, Epirus and northern Thessaly all the way to the Island of Evia, Greece (Stevanović and Jakovljević, 2014). In Bulgaria serpentine areas are smaller and scattered (Pavlova, 2010), distributed in Soutwestern \& Central Bulgaria (Stevanović et al., 2003). It should be emphasized that the floristic opulence of these habitats increases with the serpentinites of western and central Serbia, over Albania to northern Pindo and the Evia Island, Greece (Stevanović and Jakovljević, 2014).

In the territory of Serbia the largest serpentine surfaces are located in its western and central regions, as well as in Kosovo and Metohija territory, covering about 250000 ha (Stevanović et al., 2003). These serpentine zones are distinctly delimited from the adjacent surrounding regions with a different geological substratum (e.g., limestone) (Dudić et al., 2007). Kosovo, as a part of the Balkan, hosts an ultramafic area of 487 $\mathrm{km}^{2}$ within its territory (Salihaj et al., 2018). The largest complexes of serpentine in Kosovo region are found in the valley of the Ibar River and the same are continued in a discontinuous chain through Koznica Mt. and Goleš Mt. to the southwest of Kosovo (Krasniqi and Millaku, 2007). The Ibar serpentine massif stretches along the middle course of the Ibar River and represents a link in the extension chain of the serpentines in Bosnia-Zlatibor and the Ibar gorge-Albania direction (Prodanović et al., 2008). The identified age ranges from post-trias to lower jurassic.

The serpentine terrains of northeastern Kosovo, i.e. the middle stream of the Ibar river, have been rarely visited and floristically researched, which resulted in scarce scientific literature (Pavlović, 1967; Rexhepi, 1979, 1992; Krasniqi et al., 1981; Ranđelović et al., 1982; Krivošej et al., 1993, 1995-1998; Tatić and Krivošej, 1997). A more thorough exploration of this area was started in 2003 by Prodanović, whose work resulted in publishing new chorological data on the flora of Kosovo and Metohija, and entire Serbia (Prodanović, 2007; Prodanović et al., 2004, 2008, 2010, 2012, 2013, 2018; Krivošej et al., 2003, 2011, 2013). However, a comprehensive study involving all vascular serpentine flora in the Ibar valley has not been published so far.

This paper presents a synthesis of all research studies performed in these area, both literature data and intensive field study data, starting from 2003 up to the present days. Its aim is to compile a complete checklist of floristic diversity of this area, analyse it on taxonomical, ecological and phytogeographical base and to compare the flora of two serpentinite regions in central Kosovo and central Serbia. It would be a significant scientific contribution to taxonomic and ecological studies of flora in whole Serbia. 
Only a good knowledge of floristic diversity may initiate the procedures that may lead to preserving and protecting rare and endangered and internationally important species in these areas.

\section{Materials and Methods}

\section{Study area}

The intensive research studies of serpentine terrain in middle stream of the Ibar river started in March 2003 and continued till today. The researched area covers terrains in the Ibar river canyon north of Kosovska Mitrovica towards the administrative boundary of Kosovo and Metohija and central Serbia and a $50 \mathrm{~km}$ long village area of Donje Jarinje (Fig. 1). It should be emphasized that this highway more or less follows the Ibar river course. The actual length of the investigated area, due to naturally winding river course, is much bigger.

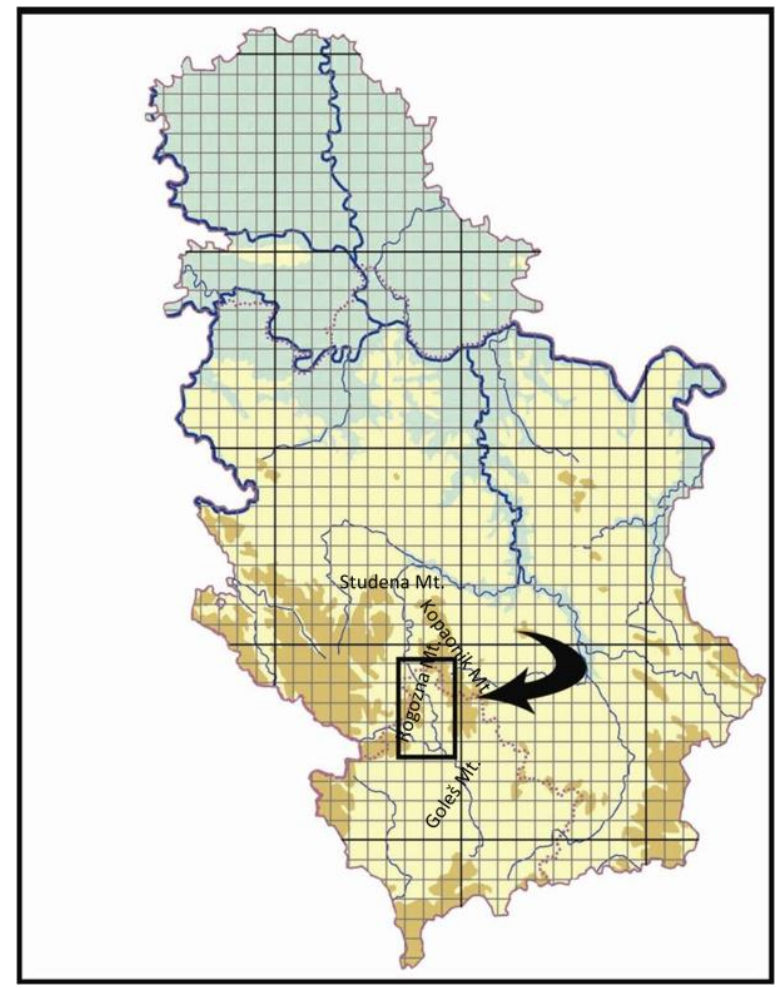

Figure 1. Geographical position of investigated area in Serbia (UTM 10x10 sq km)

The geographic relief of the Ibar river middle stream valley may be characterized as mountainous region - the Mountains of Rogozna, Kopaonik and Mokra raise up in the direction of the Ibar river canyon and they provide the main landscape characteristics to Kosovska Mitrovica basin. The altitude on the researched terrain varies between 500 and 900 meters above sea level. The climate of the Ibar river middle steam is defined as moderate -continental. The average air temperature is $10.2{ }^{\circ} \mathrm{C}$. The researched area belongs to the zone with limited precipitation rate, around $614 \mathrm{~mm}$.

The researched area in north Kosovo and Metohija covers the position between the Mesian and Illyrian provinces, since the Ibar river represents the farthest eastern 
boundary of the Illyrian province. Due to this herbal-geographic position complex floristic and vegetation relations occur.

\section{Data collection}

The floral material was collected in the wider area on both sides of the river, through the gorge. The collected material was processed on usual ways for herbariums and stored in the Herbarium of the Institute for Natural Conservation of Serbia (a department in Belgrade) and some specimens are stored in the Herbarium of the Institute of Botany and Botanical Garden "Jevremovac", University of Belgrade (BEOU). The contemporary literature has been used for plant determination. The nomenclature used for all the registered species in the area under investigation was adjusted to comply with Euro+Med Plantbase (2006) and The Plant List (2013). The floristic catalogue is arranged in alphabetical order of families and genera.

The floristic elements for phytogeographic analyses have been processed and analyzed in line with herbal-geographic classification of Stevanović (1992a). The well-known Raunkiaer system (1934) amended by Stevanović (1992b) for Serbian conditions, has been used for the classification of life forms. Checklist of Convention on International Trade in Endangered Species of Wild Fauna and Flora (CITES), as well as International Union for Conservation of Nature (IUCN) were used to determine the category of internationally significant and endangered species.

For the analysis of the similarity of flora in the researched area in the Ibar river valley and Studena planina/Mt. Studena and Goleš Mt., as parts of the Ibar serpentine massive, Sørensen's (1948) similarity index was used.

\section{Results and Discussion}

\section{Taxonomic analysis of the flora}

Biologically, serpentine sites frequently host a depauperate flora compared to the surrounding regions (Obratov-Petković et al., 2006). Serpentine habitats are important centres for floristic differentiation and speciation (Stevanović et al., 2003) distinguished by high number of endemics. Considering the fact that the serpentine terrains with the insufficiently developed soil and unfavourable water and mineral regime, and high levels of magnesium, iron, nickel and chrome are "inhospitable" for floral development, the total number of 882 identified taxa in the investigated area still show floral treasure (Prodanović et al., 2008).

During a 16-year research of serpentine terrain flora of the Ibar river middle-stream valley, 882 taxa (species, subspecies) has been identified, which represent about $25 \%$ of total flora of Serbia. They have been grouped into 83 families and 386 genera (Table 1). Class Polypodiopsida is presented by 8 families and 13 taxa. Only two species of Pinopsida can be found. Floristically speaking, the richest class is Magnoliopsida with 867 taxa. The greatest number of taxa is noted in family Compositae (105), followed by Leguminosae (86), Poaceae (66), Lamiaceae (51), Brassicaceae (50), Caryophyllaceae (49) which coincides with the most numerous families in the flora of entire Serbia (Stevanović et al., 1995). 


$$
-7293-
$$

Table 1. Catalogue of the vascular plants observed in the serpentine terrains of Kosovo's section of the Ibar river valley

\begin{tabular}{|c|c|c|}
\hline CLASS/ FAMILY/Species & Source/Literature & Common English names \\
\hline \multicolumn{3}{|l|}{ POLYPODIOPSIDA } \\
\hline \multicolumn{3}{|l|}{ ASPLENIACEAE } \\
\hline $\begin{array}{c}\text { Asplenium adiantum-nigrum } \\
\text { subsp. serpentini (Tausch) Heuf }\end{array}$ & Prodanović, 2007 & black spleenwort \\
\hline $\begin{array}{l}\text { Asplenium ceterach } \mathrm{L} \text {. } \\
\text { subsp. ceterach }\end{array}$ & Prodanović, 2007 & rustyback \\
\hline Asplenium ruta-muraria $\mathrm{L}$. & Prodanović, 2007 & wall-rue \\
\hline Asplenium trichomanes L. & Prodanović, 2007 & maidenhair spleenwort \\
\hline \multicolumn{3}{|l|}{ CYSTOPTERIDACEAE } \\
\hline Cystopteris fragilis (L.) Bernh. & Prodanović, 2007 & brittle bladder-fern \\
\hline \multicolumn{3}{|l|}{ DENNSTAEDTIACEAE } \\
\hline Pteridum aquilinum (L.) Kuhn & Prodanović, 2007 & bracken \\
\hline \multicolumn{3}{|l|}{ DRYOPTERIDACEAE } \\
\hline Dryopteris filix-mas (L.) Schott & Prodanović, 2007 & \\
\hline \multicolumn{2}{|l|}{ EQUISETACEAE } & male-fern \\
\hline Equisetum arvense L. & Prodanović, 2007; Krivošej et al., 2013 & field horsetail \\
\hline Equisetum palustre L. & Prodanović, 2007 & marsh horsetail \\
\hline \multicolumn{3}{|l|}{ OPHIOGLOSSACEAE } \\
\hline Ophioglossum vulgatum L. & Krivošej et al., 2013 & adder's tongue \\
\hline \multicolumn{3}{|l|}{ POLYPODIACEAE } \\
\hline Polypodium vulgare $\mathrm{L}$. & Prodanović, 2007 & polypody \\
\hline \multicolumn{3}{|l|}{ PTERIDACEAE } \\
\hline Paraceterach marantae (L.) R.M. Tryon & Rexhepi, 1979; Prodanović, 2007 & \\
\hline Cheilanthes persica (Bory) Mett. ex Kuhn & Krivošej et al., 2003; Prodanović, 2007 & \\
\hline \multicolumn{3}{|c|}{ PINOPSIDA } \\
\hline \multicolumn{3}{|l|}{ CUPRESSACEAE } \\
\hline Juniperus communis L. & Prodanović, 2007 & common juniper \\
\hline Juniperus oxycedrus L. & $\begin{array}{c}\text { Pavlović, 1967; Rexhepi, 1979; Rexhepi } \\
\text { 1992; Prodanović, 2007 }\end{array}$ & $\begin{array}{c}\text { western prickly juniper, cade } \\
\text { juniper }\end{array}$ \\
\hline \multicolumn{3}{|l|}{ MAGNOLIOPSIDA } \\
\hline \multicolumn{3}{|l|}{ ADOXACEAE } \\
\hline Adoxa moschatellina L. & Prodanović, 2007 & moschatel \\
\hline Viburnum lantana $\mathrm{L}$. & Prodanović, 2007 & wayfaring tree \\
\hline \multicolumn{3}{|l|}{ AMARANTHACEAE } \\
\hline Amaranthus albus $\mathrm{L}$. & Prodanović, 2007 & tumble pigweed \\
\hline Amaranthus blitoides S. Watson & Prodanović, 2007 & mat amaranth \\
\hline Amaranthus retroflexus $\mathrm{L}$. & Prodanović, 2007 & redroot pigweed \\
\hline Chenopodium bonus-henricus L. & Prodanović, 2007 & poor-man's asparagus \\
\hline Chenopodium hybridum L. & Prodanović, 2007 & goosefoot \\
\hline $\begin{array}{c}\text { Chenopodium opulifolium Schrad. ex } \\
\text { W.D.J. Koch \& Ziz } \\
\end{array}$ & Prodanović, 2007 & grey goosefoot \\
\hline Chenopodium polyspermum $\mathrm{L}$. & Prodanović, 2007 & manyseed goosefoot \\
\hline $\begin{array}{c}\text { Dysphania botrys (L.) Mosyakin \& } \\
\text { Clemants }\end{array}$ & Prodanović, 2007 & jerusalem oak goosefoot \\
\hline Polycnemum majus A. Braun & Prodanović, 2007 & giant needleleaf \\
\hline \multicolumn{3}{|l|}{ AMARYLLIDACEAE } \\
\hline $\begin{array}{c}\text { Allium carinatum } \\
\text { subsp. pulchellum (G.Don.) Bonnier \& } \\
\text { Layens } \\
\end{array}$ & Prodanović, 2007 & witch's garlic \\
\hline Allium flavum $\mathrm{L}$. & $\begin{array}{c}\text { Pavlović, 1967; Prodanović et al., 2004; } \\
\text { Prodanović, } 2007\end{array}$ & small yellow onion \\
\hline Allium moschatum L. & Prodanović, 2007 & wild onion \\
\hline Allium pendulinum Ten. & Prodanović, 2007 & italian garlic \\
\hline
\end{tabular}




\begin{tabular}{|c|c|c|}
\hline CLASS/ FAMILY/Species & Source/Literature & Common English names \\
\hline Allium scorodoprasum $\mathrm{L}$. & Pavlović, 1967; Prodanović, 2007 & rocambole \\
\hline Allium sphaerocephalum L. & Prodanović, 2007 & round-headed leek \\
\hline Galanthus nivalis L. & Prodanović, 2007 & snowdrop \\
\hline \multicolumn{3}{|l|}{ ANACARDIACEAE } \\
\hline Cotinus coggygria Scop. & $\begin{array}{l}\text { Pavlović, 1967; Rexhepi, 1979; } \\
\text { Prodanović, } 2007\end{array}$ & smoke tree \\
\hline \multicolumn{3}{|l|}{ APIACEAE } \\
\hline Aegopodium podagraria L. & Prodanović, 2007; Krivošej et al., 2013 & goutweed \\
\hline Anthriscus caucalis M.Bieb. & Prodanović, 2007 & bur-chervil \\
\hline Anthriscus cerefolium (L.) Hoffm. & Prodanović, 2007 & garden chervil \\
\hline Anthriscus sylvestris (L.) Hoffm. & Prodanović, 2007 & cow parsley, wild chervil \\
\hline Bifora radians $\mathrm{M}$. Bieb. & Prodanović, 2007 & wild bishop \\
\hline Bupleurum praealtum $\mathrm{L}$. & Prodanović, 2007 & hare's-ears \\
\hline Bupleurum rotundifolium $\mathrm{L}$. & Prodanović, 2007 & round-leaved thoroughwax \\
\hline Bupleurum tenuissimum $\mathrm{L}$. & Prodanović et al., 2013 & slender hare's-ear \\
\hline Chaerophyllum aureum $\mathrm{L}$. & Prodanović, 2007 & golden chervil \\
\hline Chaerophyllum bulbosum L. & Prodanović, 2007 & turnip rooted chervil \\
\hline Chaerophyllum temulum $\mathrm{L}$. & Prodanović, 2007 & rough chervil, \\
\hline Conium maculatum L. & Prodanović, 2007 & poison hemlock \\
\hline Daucus carota $\mathrm{L}$. & Prodanović, 2007 & wild carrot, bird's nest \\
\hline Eryngium campestre L. & Rexhepi, 1979; Prodanović, 2007 & field eryngo \\
\hline Eryngium palmatum Pančić \& Vis. & Prodanović, 2007 & blue eryngo, flat sea holly \\
\hline Eryngium serbicum Pančić & $\begin{array}{c}\text { Prodanović, 2007; Prodanović et al., } \\
2008\end{array}$ & serbian sea holly \\
\hline Falcaria vulgaris Bernh. & Prodanović, 2007 & sickleweed \\
\hline Foeniculum vulgare Mill. & Prodanović, 2007 & sweet fennel \\
\hline Heracleum sphondylium $\mathrm{L}$. & Prodanović, 2007 & common hogweed \\
\hline Laser trilobum (L.) Borkh. & Prodanović, 2007 & gladich \\
\hline Laserpitium siler $\mathrm{L}$. & Prodanović, 2007 & laserwort \\
\hline $\begin{array}{l}\text { Laserpitium siler } \mathrm{L} . \\
\text { subsp. siler }\end{array}$ & Prodanović, 2007 & laserwort \\
\hline Myrrhoides nodosa (L.) Cannon & Prodanović, 2007 & sweet cicely \\
\hline Oenanthe silaifolia M.Bieb. & Prodanović, 2007; Krivošej et al., 2013 & narrow-leaved water-dropwort \\
\hline Orlaya grandiflora (L.) Hoffm. & Prodanović, 2007 & white laceflower \\
\hline Pastinaca sativa $\mathrm{L}$. & Prodanović, 2007 & parsnip \\
\hline $\begin{array}{l}\text { Pastinaca sativa } \mathrm{L} \text {. } \\
\text { subsp. urens (Godr.) }\end{array}$ & Prodanović, 2007 & wild parsnip \\
\hline Peucedanum alsaticum $\mathrm{L}$. & Prodanović, 2007 & hog's fennel \\
\hline $\begin{array}{l}\text { Peucedanum austriacum (Jacq.) W.D.J. } \\
\text { Koch }\end{array}$ & Prodanović, 2007 & giant hog's fennel \\
\hline $\begin{array}{l}\text { Peucedanum cervaria (L.) Cusson ex } \\
\text { Lapeyr. }\end{array}$ & Prodanović, 2007 & hart's word \\
\hline Peucedanum officinale L. & Prodanović, 2007 & marsh hog's fennel \\
\hline Peucedanum oreoselinum (L.) Moench & Prodanović, 2007 & mountain hog's Fennel \\
\hline Pimpinella saxifraga $\mathrm{L}$. & Prodanović, 2007 & burnet-saxifrage, \\
\hline Physospermum cornubiense (L.) DC. & Prodanović, 2007 & bladderseed \\
\hline Scandix pecten-veneris $\mathrm{L}$. & Prodanović, 2007 & Venus' comb \\
\hline Seseli pallasii Besser & Prodanović, 2007 & \\
\hline $\begin{array}{l}\text { Seseli peucedanoides (M.Bieb.) Koso.- } \\
\text { Pol. }\end{array}$ & Prodanović, 2007 & \\
\hline Seseli rigidum Waldst. \& Kit. & Prodanović, 2007 & \\
\hline $\begin{array}{l}\text { Seseli rigidum Waldst. \& Kit. } \\
\text { subsp. rigidum }\end{array}$ & Prodanović, 2007 & \\
\hline Sium latifolium $\mathrm{L}$. & Prodanović, 2007 & water hemlock \\
\hline Smyrnium perfoliatum $\mathrm{L}$. & Prodanović, 2007 & perfoliate alexanders \\
\hline Torilis japonica (Houtt.) DC. & Prodanović, 2007 & erect hedgeparsley \\
\hline Trinia glauca (L.) Dumort. & Prodanović, 2007 & honewort \\
\hline
\end{tabular}




$$
\text { - } 7295 \text { - }
$$

\begin{tabular}{|c|c|c|}
\hline CLASS/ FAMILY/Species & Source/Literature & Common English names \\
\hline \multicolumn{3}{|l|}{ subsp. glauca } \\
\hline \multicolumn{3}{|l|}{ APOCYNACEAE } \\
\hline Vinca major $\mathrm{L}$. & Prodanović, 2007 & greater periwinkle \\
\hline Vincetoxicum hirundinaria Medik. & Prodanović, 2007 & white swallow-wort \\
\hline \multicolumn{3}{|l|}{ ARACEAE } \\
\hline Arum maculatum $\mathrm{L}$. & Prodanović, 2007 & cuckoo pint \\
\hline \multicolumn{3}{|l|}{ ARALIACEAE } \\
\hline Hedera helix $\mathrm{L}$. & Prodanović, 2007 & english ivy \\
\hline \multicolumn{3}{|l|}{ ARISTOLOCHIACEAE } \\
\hline Aristolochia clematitis $\mathrm{L}$. & Prodanović, 2007 & birthwort \\
\hline Asarum europaeum L. & Prodanović, 2007 & wild ginger \\
\hline \multicolumn{3}{|l|}{ ASPARAGACEAE } \\
\hline Anthericum liliago $\mathrm{L}$. & Pavlović, 1967; Prodanović, 2007 & lily \\
\hline Asparagus officinalis $\mathrm{L}$. & Prodanović, 2007 & sparrow grass \\
\hline Asparagus tenuifolius Lam. & Rexhepi, 1979 & \\
\hline Convallaria majalis $\mathrm{L}$. & Prodanović, 2007 & lily of the valleys \\
\hline Leopoldia comosa (L.) Parl. & Prodanović, 2007 & tassel hyacinth \\
\hline Muscari racemosum Mill. & Prodanović, 2007 & starch grape hyacinth \\
\hline Ornithogalum gussonei Ten. & Prodanović, 2007 & star-of-Bethlehem \\
\hline Ornithogalum pyramidale L. & Prodanović, 2007 & pyramidal star-of-Bethlehem \\
\hline Ornithogalum refractum Kit. ex Schltdl. & Prodanović, 2007 & star-of-Bethlehem, \\
\hline Ornithogalum umbellatum $\mathrm{L}$. & Prodanović, 2007 & garden star-of-Bethlehem \\
\hline Polygonatum hirtum (Bosc ex Poir) Pursh & Prodanović, 2007 & king Solomon's-seal \\
\hline Polygonatum odoratum (Mill.) Druce & Prodanović, 2007 & fragrant Solomon's seal \\
\hline Scilla bifolia $\mathrm{L}$. & Prodanović, 2007 & twin leaf squill \\
\hline \multicolumn{3}{|l|}{ BERBERIDACEAE } \\
\hline $\begin{array}{l}\text { Berberis vulgaris L. } \\
\text { f. vulgaris }\end{array}$ & Prodanović, 2007 & common barberry \\
\hline Epimedium alpinum $L$. & Prodanović et al., 2013 & alpine barrenwort \\
\hline \multicolumn{3}{|l|}{ BETULACEAE } \\
\hline Alnus glutinosa (L.) Gaertn. & Prodanović, 2007; Krivošej et al., 2013 & european black alder \\
\hline Carpinus betulus $\mathrm{L}$. & Prodanović, 2007 & common hornbeam \\
\hline Carpinus orientalis Mill. & $\begin{array}{c}\text { Pavlović, 1967; Prodanović et al., 2004; } \\
\text { Prodanović, } 2007\end{array}$ & oriental hornbeam \\
\hline Corylus avellana $\mathrm{L}$. & Prodanović, 2007; Krivošej et al., 2013 & hazel \\
\hline Ostrya carpinifolia Scop. & \begin{tabular}{|c|} 
Prodanović et al., 2004; Prodanović, \\
2007
\end{tabular} & hop-hornbeam \\
\hline \multicolumn{3}{|l|}{ BORAGINACEAE } \\
\hline Anchusa azurea Mill. & Prodanović, 2007 & bugloss \\
\hline Anchusa officinalis L. & Prodanović, 2007 & common bugloss, alkanet \\
\hline $\begin{array}{l}\text { Anchusa officinalis } \mathrm{L} \text {. } \\
\text { subsp. officinalis }\end{array}$ & Prodanović, 2007 & common bugloss, alkanet \\
\hline Asperugo procumbens $\mathrm{L}$. & Prodanović, 2007 & german-madwort \\
\hline Buglossoides arvensis (L.) I.M. Johnst. & Prodanović, 2007 & corn gromwell \\
\hline $\begin{array}{c}\text { Buglossoides purpurocaerulea (L.) I.M. } \\
\text { Johnst. }\end{array}$ & Prodanović, 2007 & purple gromwell \\
\hline Cerinthe minor $\mathrm{L}$. & Prodanović, 2007 & honeyworts \\
\hline Cynoglossum creticum Mill. & Prodanović, 2007 & blue houndstonge \\
\hline Cynoglossum officinale L. & Prodanović, 2007 & houndstongue \\
\hline Echium rubrum Forssk. & $\begin{array}{l}\text { Rexhepi, 1979; Krivošej et al., 1993; } \\
\text { Prodanović, } 2007\end{array}$ & viper's bugloss rubrum \\
\hline Echium italicum L. & Prodanović, 2007 & italian bugloss \\
\hline Echium vulgare L. & Prodanović, 2007 & common vipersbugloss \\
\hline Halacsya sendtneri (Boiss.) Dörfl. & $\begin{array}{l}\text { Pavlović, 1967; Rexhepi, 1979; } \\
\text { Ranđelović et al., 1982; Prodanović, } \\
\text { 2007; Prodanović et al., } 2008\end{array}$ & halacsya \\
\hline Heliotropium europaeum L. & Prodanović, 2007 & european turn-sole \\
\hline
\end{tabular}




$$
\text { - } 7296 \text { - }
$$

\begin{tabular}{|c|c|c|}
\hline CLASS/ FAMILY/Species & Source/Literature & Common English names \\
\hline Lappula squarrosa (Retz.) Dumort. & Prodanović, 2007 & bur forget-me-not \\
\hline Myosotis arvensis (L.) Hill. & Prodanović, 2007 & field forget-me-not \\
\hline Myosotis discolor Pers. & Prodanović, 2007 & changing forget-me-not \\
\hline Myosotis sparsiflora J.K.Mikan ex Pohl. & Prodanović, 2007 & forget-me-not \\
\hline Nonnea pulla DC. & Prodanović, 2007 & monkswort \\
\hline Onosma montana $\mathrm{Sm}$. & Prodanović, 2007 & onosma \\
\hline $\begin{array}{l}\text { Pulmonaria mollissima Wulfen ex } \\
\text { Hornem. }\end{array}$ & Prodanović, 2007 & lungwort \\
\hline Pulmonaria officinalis $\mathrm{L}$. & Prodanović, 2007 & common lungwort \\
\hline Symphytum tuberosum $\mathrm{L}$. & Prodanović, 2007 & tuberous comfrey \\
\hline \multicolumn{3}{|l|}{ BRASSICACEAE } \\
\hline $\begin{array}{c}\text { Aethionema saxatile } \\
\text { subsp. graecum (Boiss. \& Spruner) Hayek }\end{array}$ & Rexhepi, 1979; Prodanović, 2007 & burnt candytuft \\
\hline $\begin{array}{c}\text { Aethionema saxatile (L.) R.Br. } \\
\text { subsp. saxatile }\end{array}$ & Prodanović, 2007 & burnt candytuft \\
\hline $\begin{array}{c}\text { Alliaria petiolata (M.Bieb.) Cavara \& } \\
\text { Grande }\end{array}$ & Prodanović, 2007 & garlic mustard \\
\hline Alyssum alyssoides (L.) L. & Prodanović, 2007 & yellow alyssum \\
\hline Alyssum markgrafii O.E.Schulz. & $\begin{array}{c}\text { Pavlović, 1967; Rexhepi, 1979; Krivošej } \\
\text { et al., 1993; Prodanović, 2007; } \\
\text { Prodanović et al., 2008; }\end{array}$ & \\
\hline $\begin{array}{l}\text { Alyssum montanum L. } \\
\text { subsp. serbicum Novák } \\
\text { f. macrophyllum Novák }\end{array}$ & $\begin{array}{c}\text { Pavlović, 1967; Prodanović, 2007; } \\
\text { Prodanović et al., } 2008\end{array}$ & mountain alyssum \\
\hline $\begin{array}{l}\text { Alyssum montanum L. } \\
\text { subsp. serbicum Novák } \\
\text { f. microphyllum Novák }\end{array}$ & Pavlović, 1967; Prodanović, 2007 & mountain alyssum \\
\hline $\begin{array}{l}\text { Alyssum montanum L. } \\
\text { subsp. serbicum Novák } \\
\text { f. serbicum }\end{array}$ & Pavlović, 1967; Prodanović, 2007 & mountain alyssum \\
\hline Alyssum wierzbickii Heuff.? & Prodanović, 2007 & alpine alyssum \\
\hline Arabidopsis arenosa (L.) Lawalrée & $\begin{array}{c}\text { Prodanović, (unpublished, field } \\
\text { observation) }\end{array}$ & rockcres \\
\hline Arabidopsis thaliana (L.) Heynh. & Prodanović, 2007 & thale cress, mouse-ear cress \\
\hline Arabis hirsuta (L.) Scop. & Prodanović, 2007 & hairy rock-cress \\
\hline Arabis turrita $\mathrm{L}$. & Prodanović, 2007 & rockcress, \\
\hline Berteroa incana (L.) DC. & Prodanović, 2007 & hoary alyssum \\
\hline Calepina irregularis (Asso) Thell. & Prodanović, 2007 & white ballmustard \\
\hline Camelina sativa (L.) Crantz. & Prodanović, 2007 & camelina, false flax \\
\hline Capsella bursa-pastoris (L.) Medik & Prodanović, 2007 & shepherd's purse \\
\hline Cardamine bulbifera (L.) Crantz. & Prodanović, 2007 & coralroot \\
\hline Cardamine graeca $\mathrm{L}$. & Krivošej \& Prodanović, 2011 & southern bitter-cress \\
\hline Cardamine impatiens $\mathrm{L}$. & Prodanović, 2007 & narrowleaf bittercress \\
\hline Cardamine pratensis L. & Prodanović, 2007 & cuckoo flower, lady's smock \\
\hline Descurainia sophia (L.) Webb. ex Prantl & Prodanović, 2007 & flixweed \\
\hline Diplotaxis muralis (L.) DC. & Prodanović, 2007 & wall rocket \\
\hline Draba lasiocarpa Rochel & Prodanović, 2007 & whitlow-grasses \\
\hline Draba muralis L. & Prodanović, 2007 & whitlowgrass \\
\hline Erysimum cuspidatum (M. Bieb.) DC. & Prodanović, 2007 & wallflower \\
\hline Erysimum diffusum Ehrh. & Pavlović, 1967; Prodanović, 2007 & diffuse wallflowe \\
\hline Erysimum odoratum Ehrh. & $\begin{array}{c}\text { Prodanović, (unpublished, field } \\
\text { observation) }\end{array}$ & smelly wallflower \\
\hline Erysimum kuemmerlei Jáv. & Prodanović, 2007 & wallflower \\
\hline Erysimum sylvestre (Crantz) Scop. & Prodanović, 2007 & wallflower \\
\hline Fibigia clypeata (L.) Medik & $\begin{array}{c}\text { Prodanović et al., 2004; Prodanović, } \\
2007\end{array}$ & false-gypsophila ankyropetalum \\
\hline Hesperis matronalis L. & Prodanović, 2007 & mother-of-the-evening \\
\hline Isatis tinctoria $\mathrm{L}$. & Prodanović, 2007 & dyer's woad, glastum \\
\hline
\end{tabular}




$$
\text { - } 7297 \text { - }
$$

\begin{tabular}{|c|c|c|}
\hline CLASS/ FAMILY/Species & Source/Literature & Common English names \\
\hline Lepidium campestre (L.) R.Br. & Prodanović, 2007 & field pepperwort, field cress \\
\hline Lepidium draba $\mathrm{L}$. & Prodanović, 2007 & whitetop, hoary cress \\
\hline Lepidium ruderale $\mathrm{L}$. & Prodanović, 2007 & roadside pepperweed \\
\hline Myagrum perfoliatum L. & Prodanović, 2007 & bird's-eye cress, muskweed \\
\hline $\begin{array}{c}\text { Odontarrhena bertolonii } \\
\text { subsp. scutarina (Nyár) Španiel \& al. }\end{array}$ & Prodanović, 2007 & \\
\hline Rorippa amphibia (L.) Besser & Prodanović, 2007 & great yellowcress \\
\hline Rorippa austriaca (Crantz) Spach. & Prodanović, 2007 & austrian yellow-cress \\
\hline Rorippa lippizensis (Wulfen) Rchb. & Prodanović, 2007 & yellowcress \\
\hline Rorippa sylvestris (L.) Besser & Prodanović, 2007 & $\begin{array}{l}\text { creeping yellowcress, yellow } \\
\text { fieldcress }\end{array}$ \\
\hline Sisymbrium altissimum L. & Prodanović, 2007 & $\begin{array}{l}\text { tall hedge-mustard, } \\
\text { tumblemustard }\end{array}$ \\
\hline Sisymbrium loeselii L. & Prodanović, 2007 & small tumbleweed mustard \\
\hline Sisymbrium officinale (L.) Scop. & Prodanović, 2007 & hedge mustard \\
\hline Sisymbrium orientale $\mathrm{L}$. & Prodanović, 2007 & indian hedgemustard \\
\hline Sisymbrium strictissimum L. & Prodanović, 2007 & perennial rocket \\
\hline Thlaspi arvense $\mathrm{L}$. & Prodanović, 2007 & $\begin{array}{c}\text { stinkweed, bastard cress, } \\
\text { fanweed }\end{array}$ \\
\hline Thlaspi kovatsii Heuff. & Prodanović, 2007 & pennycress \\
\hline Turritis glabra L. & Prodanović, 2007 & tower rockcress, tower mustard \\
\hline \multicolumn{3}{|l|}{ CAMPANULACEAE } \\
\hline $\begin{array}{l}\text { Asyneuma canescens (Waldst. \& Kit.) } \\
\text { Griseb. \& Schenk }\end{array}$ & Prodanović, 2007 & harebells \\
\hline Asyneuma limonifolium (L.) Janch. & $\begin{array}{c}\text { Pavlović, 1967; Ranđelović et al., 1982; } \\
\text { Prodanović, } 2007\end{array}$ & harebells \\
\hline Campanula cervicaria $\mathrm{L}$. & \begin{tabular}{|c|c|} 
Prodanović, 2007 \\
\end{tabular} & bristly bellflower \\
\hline Campanula lingulata Waldst. \& Kit. & $\begin{array}{c}\text { Rexhepi, 1979; Prodanović et al., 2004; } \\
\text { Prodanović, } 2007\end{array}$ & bellflower \\
\hline Campanula persicifolia $\mathrm{L}$. & Prodanović, 2007 & peach-leaved bellflower \\
\hline Campanula rapunculoides $\mathrm{L}$. & Prodanović, 2007 & creeping bellflower \\
\hline Campanula rapunculus $\mathrm{L}$. & Prodanović, 2007 & rampion bellflower \\
\hline $\begin{array}{l}\text { Campanula rapunculus L. } \\
\text { f. montana Pančić }\end{array}$ & Prodanović, 2007 & rampion bellflower, rampion \\
\hline Campanula trachelium L. & Prodanović, 2007 & nettle-leaved bellflower \\
\hline $\begin{array}{l}\text { Legousia speculum-veneris (L.) Durande } \\
\text { ex Vill. }\end{array}$ & Prodanović, 2007 & $\begin{array}{c}\text { looking glass, large Venus's- } \\
\text { looking-glass }\end{array}$ \\
\hline \multicolumn{3}{|l|}{ CANNABACEAE } \\
\hline Humulus lupulus L. & Prodanović, 2007 & common hop \\
\hline \multicolumn{3}{|l|}{ CAPRIFOLIACEAE } \\
\hline $\begin{array}{l}\text { Cephalaria leucantha (L.) Scharad. ex } \\
\text { Roem. \& Schult. }\end{array}$ & Rexhepi, 1979; Prodanović, 2007 & giant scabious \\
\hline Knautia arvensis (L.) Coult. & Prodanović, 2007 & field scabious \\
\hline Knautia integrifolia (Honck. ex L.) Bertol & Prodanović, 2007 & whole-leaved scabious \\
\hline Lonicera caprifolium $\mathrm{L}$. & Prodanović, 2007 & $\begin{array}{l}\text { italian woodbine, } \\
\text { perfoliate honeysuckle }\end{array}$ \\
\hline Scabiosa argentea $\mathrm{L}$. & $\begin{array}{c}\text { Prodanović, (unpublished, field } \\
\text { observation) }\end{array}$ & silver scabious \\
\hline Scabiosa columbaria $\mathrm{L}$. & Rexhepi, 1979; Prodanović, 2007 & $\begin{array}{l}\text { pigeon scabious, pincushion } \\
\text { flower }\end{array}$ \\
\hline Scabiosa fumaroides Vis. \& Pančić & $\begin{array}{c}\text { Prodanović, 2007; Prodanović et al., } \\
2008 \\
\end{array}$ & \\
\hline Valeriana officinalis L. & Prodanović, 2007 & garden heliotrope, valerian \\
\hline Valeriana tuberosa L. & $\begin{array}{c}\text { Krivošej et al., 1995-1998; Prodanović, } \\
2007\end{array}$ & tuberous valerian \\
\hline Valerianella coronata (L.) DC. & Prodanović, 2007 & corn lettuce \\
\hline Valerianella dentata (L.) Pollich & $\begin{array}{c}\text { Ranđelović et al., 1982; Prodanović, } \\
2007 \\
\end{array}$ & corn salad \\
\hline Valerianella locusta (L.) Laterr. & Prodanović, 2007 & corn salad \\
\hline
\end{tabular}




\begin{tabular}{|c|c|c|}
\hline CLASS/ FAMILY/Species & Source/Literature & Common English names \\
\hline Valerianella rimosa Bastard & Prodanović, 2007 & beaked corn salad \\
\hline \multicolumn{3}{|l|}{ CARYOPHYLLACEAE } \\
\hline Agrostemma githago L. & Prodanović, 2007 & corncockle \\
\hline $\begin{array}{c}\text { Arenaria serpyllifolia } \\
\text { subsp. leptoclados (Rchb.) Nyman }\end{array}$ & Prodanović, 2007 & thyme-leaf sandwort \\
\hline Arenaria serpyllifolia L. & Prodanović, 2007 & thyme-leaf sandwort \\
\hline Cerastium brachypetalum Desp. ex Pers & Prodanović, 2007 & gray chickweed \\
\hline Cerastium decalvans Schloss. \& Vuk. & Prodanović, 2007 & balkan rozhets \\
\hline Cerastium fontanum Baumg. & Prodanović, 2007 & mouse-ear chickweed \\
\hline Cerastium pumilum Curtis & Prodanović, 2007 & $\begin{array}{c}\text { dwarf mouse-ear, european } \\
\text { chickweed }\end{array}$ \\
\hline $\begin{array}{c}\text { Cerastium pumilum } \\
\text { var. glutinosum (Čelak) E.Rico }\end{array}$ & Prodanović, 2007 & dwarf mouse-ear \\
\hline Cerastium semidecandrum L. & Prodanović, 2007 & fivestamen chickweed \\
\hline Dianthus carthusianorum L. & Prodanović, 2007 & carthusian pink \\
\hline Dianthus giganteus d'Urv & Prodanović, 2007 & pink \\
\hline Dianthus pinifolius $\mathrm{Sm}$. & Prodanović, 2007 & immediate children \\
\hline $\begin{array}{c}\text { Dianthus pinifolius } \\
\text { subsp. serbicus Wettst. }\end{array}$ & Pavlović, 1967; Prodanović, 2007 & Immediate children \\
\hline $\begin{array}{l}\text { Dianthus sylvestris Wulfen } \\
\text { subsp. sylvestris }\end{array}$ & Rexhepi, 1979; Prodanović, 2007 & wood pink \\
\hline Herniaria glabra L. & Prodanović, 2007 & smooth rupturewort \\
\hline Herniaria hirsuta L. & Prodanović, 2007 & hairy rupturewort. \\
\hline Herniaria incana Lam. & Prodanović, 2007 & grey rupturewort \\
\hline Holosteum umbellatum L. & Prodanović, 2007 & jagged chickweed \\
\hline Minuartia glomerata (M.Bieb.) Degen. & $\begin{array}{c}\text { Prodanović et al., 2004; Prodanović, } \\
2007\end{array}$ & stitchwort \\
\hline Minuartia hamata (Hausskn.) Mattf. & Prodanović, 2007 & sandwort \\
\hline Minuartia hirsuta (M.Bieb) Hand.-Mazz. & Rexhepi, 1979; Prodanović, 2007 & hairy sandwort \\
\hline Minuartia setacea (Thuill.) Hayek & Prodanović, 2007 & sandwort \\
\hline Minuartia verna (L.) Hiern & Prodanović, 2007 & leadwort \\
\hline Moehringia trinervia (L.) Clairv. & Prodanović, 2007 & $\begin{array}{l}\text { apetalous sandwort, three- } \\
\text { nerved sandwort }\end{array}$ \\
\hline $\begin{array}{c}\text { Petrorhagia illyrica } \\
\text { subsp. haynaldiana (Janka) P.W. Ball \& } \\
\text { Heywood } \\
\end{array}$ & Pavlović, 1967; Prodanović, 2007 & \\
\hline $\begin{array}{c}\text { Petrorhagia illyrica (Ard.) P.W.Ball \& } \\
\text { Heyood. }\end{array}$ & Pavlović, 1967; Prodanović, 2007 & \\
\hline $\begin{array}{c}\text { Petrorhagia prolifera (L.) P.W. Ball. \& } \\
\text { Heywood }\end{array}$ & Rexhepi, 1979; Prodanović, 2007 & proliferous pink \\
\hline Petrorhagia saxifraga (L.) Link. & $\begin{array}{c}\text { Pavlović, 1967; Rexhepi, 1979; } \\
\text { Prodanović et al., 2004; Prodanović, } \\
2007\end{array}$ & tunic flower, coat flower \\
\hline Saponaria glutinosa M. Bieb. & Prodanović, 2007 & soapwort \\
\hline Saponaria officinalis L. & Prodanović, 2007 & common soapwort, bouncing-bet \\
\hline $\begin{array}{c}\text { Scleranthus annuus } \\
\text { subsp. polycarpos (L.) Bonnier \& Layens }\end{array}$ & Prodanović, 2007 & annual knawel \\
\hline $\begin{array}{c}\text { Scleranthus perennis } \\
\text { subsp. dichotomus (Schur) Nyman }\end{array}$ & Prodanović, 2007 & perennial knawel \\
\hline $\begin{array}{c}\text { Silene latifolia } \\
\text { subsp. alba (Mill.) Greuter \& Burdet }\end{array}$ & Prodanović, 2007 & bladder campion \\
\hline Silene armeria $\mathrm{L}$. & Prodanović, 2007 & sweet William catchfly \\
\hline Silene baccifera (L.) Roth. & Prodanović, 2007 & campion \\
\hline Silene bupleuroides L. & Prodanović, 2007 & campion \\
\hline Silene conica $\mathrm{L}$. & Prodanović, 2007 & striped corn catchfly \\
\hline Silene coronaria (Desr.) Clairv. ex Rchb. & Prodanović, 2007 & rose campion \\
\hline Silene flos-cuculi (L.) Greuter \& Burdet & Prodanović, 2007 & ragged-robin \\
\hline Silene italica (L.) Pers. & Prodanović, 2007 & italian catchfly \\
\hline
\end{tabular}




$$
\text { - } 7299 \text { - }
$$

\begin{tabular}{|c|c|c|}
\hline CLASS/ FAMILY/Species & Source/Literature & Common English names \\
\hline Silene nutans L. & Prodanović, 2007 & nottingham catchfly \\
\hline Silene otites (L.) Wibel. & $\begin{array}{c}\text { Prodanović, 2007, Ranđelović et al., } \\
1982 \\
\end{array}$ & spanish catchfly \\
\hline Silene viscaria (L.) Jess. & Prodanović, 2007 & sticky catchfly \\
\hline Silene vulgaris (Moench) Garcke & Prodanović, 2007 & bladder campion \\
\hline Stellaria aquaticua (L.) Scop. & Prodanović, 2007 & giant chickweed \\
\hline Stellaria graminea $\mathrm{L}$. & Prodanović, 2007; Krivošej et al., 2013 & grassleaved stichwort \\
\hline Stellaria holostea $\mathrm{L}$. & Prodanović, 2007 & greater stitchwort \\
\hline Stellaria media (L.) Vill. & Prodanović, 2007 & common chickweed \\
\hline Stellaria nemorum L. & Prodanović, 2007 & nodding chickweed \\
\hline \multicolumn{3}{|l|}{ CELASTRACEAE } \\
\hline $\begin{array}{c}\text { Euonymus europaeus L. } \\
\text { var. europaeus } \\
\text { f. angustifolia (Schultz) Rony }\end{array}$ & Prodanović, 2007 & european spindle \\
\hline $\begin{array}{l}\text { Euonymus europaeus L. } \\
\text { var. grandifolia Form. } \\
\text { f. scaberula (Beck) Jovanović }\end{array}$ & Prodanović, 2007 & european spindle \\
\hline Euonymus latifolius (L.) Mill. & Prodanović, 2007 & broadleaf spindle \\
\hline Euonymus verrucosus Scop. & Prodanović, 2007 & spindle tree \\
\hline \multicolumn{3}{|l|}{ CERATOPHYLLACEAE } \\
\hline Ceratophyllum submersum L. & Prodanović, 2007 & soft hornwort \\
\hline \multicolumn{3}{|l|}{ CISTACEAE } \\
\hline Fumana bonapartei Maire et Petitm. & $\begin{array}{l}\text { Pavlović, 1967; Rexhepi, 1979; } \\
\text { Ranđelović et al., 1982; Prodanović, } \\
\text { 2007; Prodanović et al., } 2008\end{array}$ & needle sunrose \\
\hline $\begin{array}{l}\text { Fumana procumbens (Dunal) Gren. \& } \\
\text { Godr. }\end{array}$ & $\begin{array}{l}\text { Prodanović et al., 2004; Prodanović, } \\
2007\end{array}$ & sprawling needle sunrose \\
\hline $\begin{array}{l}\text { Helianthemum nummularium (L.) Mill. } \\
\text { subsp. nummularium }\end{array}$ & $\begin{array}{c}\text { Prodanović et al., 2004; Prodanović, } \\
2007 \\
\end{array}$ & common rock-rose \\
\hline Helianthemum salicifolium (L.) Mill. & Prodanović, 2007 & rock rose, sunrose \\
\hline \multicolumn{3}{|l|}{ CLUSIACEAE } \\
\hline Hypericum barbatum Jacq. & Prodanović, 2007 & bearded St. John's wort \\
\hline Hypericum hirsutum L. & Prodanović, 2007 & hairy St John's-wort \\
\hline Hypericum perforatum $\mathrm{L}$. & Rexhepi, 1979; Prodanović, 2007 & perforate St. John's wort \\
\hline Hypericum rumeliacum Boiss. & Prodanović, 2007 & St. John's wort \\
\hline \multicolumn{3}{|l|}{ COLCHICACEAE } \\
\hline Colchicum autumnale L. & $\begin{array}{c}\text { Prodanović et al., 2004; Prodanović, } \\
\text { 2007; Krivošej et al., } 2013\end{array}$ & autumn crocus, meadow saffron \\
\hline \multicolumn{3}{|l|}{ COMPOSITAE } \\
\hline Achillea coarctata Poir. & Prodanović, 2007 & yarrow \\
\hline $\begin{array}{l}\text { Achillea collina (Becker ex Rchb.f.) } \\
\text { Heimerl. }\end{array}$ & Prodanović, 2007 & yarrow \\
\hline Achillea critmifolia Waldst. \& Kit. & Prodanović, 2007 & yarrow \\
\hline Achillea millefolium $\mathrm{L}$. & Prodanović, 2007 & milfoil, common yarrow \\
\hline Achillea pseudopectinata Janka & Prodanović, 2007 & yarrow \\
\hline Ambrosia artemisiifolia $\mathrm{L}$. & Prodanović, 2007 & $\begin{array}{c}\text { common ragweed, annual } \\
\text { ragweed }\end{array}$ \\
\hline Anthemis arvensis $\mathrm{L}$. & Prodanović, 2007 & corn chamomile, mayweed \\
\hline Arctium lappa $\mathrm{L}$. & Prodanović, 2007 & greater burdock \\
\hline Artemisia absinthium L. & Prodanović, 2007 & absinth wormwood \\
\hline Artemisia alba Turra & Prodanović, 2007 & white wormwood \\
\hline Artemisia campestris L. & Prodanović, 2007 & common sagewort \\
\hline Artemisia scoparia Waldst. \& Kitam. & Prodanović, 2007 & virgate wormwood \\
\hline Artemisia vulgaris $\mathrm{L}$. & Prodanović, 2007 & common mugwort \\
\hline Bellis perennis L. & Prodanović, 2007; Krivošej et al., 2013 & common daisy \\
\hline Carduus candicans Waldst. \& Kit. & Prodanović, 2007 & thistle \\
\hline Carduus nutans L. & Prodanović, 2007 & musk thistle \\
\hline
\end{tabular}




$$
-7300 \text { - }
$$

\begin{tabular}{|c|c|c|}
\hline CLASS/ FAMILY/Species & Source/Literature & Common English names \\
\hline Carlina acaulis L. & Prodanović, 2007 & $\begin{array}{l}\text { stemless carline thistle, dwarf } \\
\text { carline thistle }\end{array}$ \\
\hline Carthamus lanatus L. & Prodanović, 2007 & $\begin{array}{c}\text { woolly carthamus, } \\
\text { woolly safflower }\end{array}$ \\
\hline Centaurea jacea L. & Prodanović, 2007 & brown knapweed \\
\hline Centaurea orientalis L. & Prodanović, 2007 & knapweed \\
\hline Centaurea phrygia L. & Rexhepi, 1979; Prodanović, 2007 & wig knapweed \\
\hline $\begin{array}{l}\text { Centaurea scabiosa L. } \\
\text { subsp. fritschii (Hayek) Hayek }\end{array}$ & Prodanović, 2007 & greater knapweed \\
\hline $\begin{array}{l}\text { Centaurea scabiosa L. } \\
\text { subsp. spinulosa (Spreng.) Arcang }\end{array}$ & Prodanović, 2007 & $\begin{array}{c}\text { scabious knapweed, greater } \\
\text { knapweed } \\
\end{array}$ \\
\hline Centaurea solstitialis L. & Prodanović, 2007 & yellow star-thistle \\
\hline $\begin{array}{c}\text { Centaurea stoebe L. } \\
\text { subsp. austrialis (Pančić ex A.Kern) } \\
\text { Greuter }\end{array}$ & $\begin{array}{l}\text { Pavlović, 1967; Rexhepi, 1979; } \\
\text { Prodanović, } 2007\end{array}$ & spotted knapweed \\
\hline Centaurea stoebe $\mathrm{L}$. & Prodanović, 2007 & $\begin{array}{l}\text { panicled knapweed, spotted } \\
\text { knapweed }\end{array}$ \\
\hline Chondrilla juncea $\mathrm{L}$. & $\begin{array}{c}\text { Prodanović et al., 2004; Prodanović, } \\
2007 \\
\end{array}$ & rush skeletonweed, nakedweed \\
\hline Cichorium intybus L. & Prodanović, 2007 & chicory \\
\hline Cirsium arvense (L.) Scop. & Prodanović, 2007 & creeping thistle \\
\hline Cirsium creticum (Lam.) Urv. & Prodanović, 2007 & bull thistle \\
\hline Cirsium lanceolatum (L.) Hill. & Prodanović, 2007 & bull thistle, common thistle \\
\hline Cota austriaca (Jacq.) Sch.Bip. & Prodanović, 2007 & austrian chamomile \\
\hline Cota tinctoria (L.) J.Gay. & Prodanović, 2007 & $\begin{array}{l}\text { golden marguerite, yellow } \\
\text { chamomile }\end{array}$ \\
\hline Crepis biennis Lapeyr. & Prodanović, 2007 & rough Hawksbeard \\
\hline $\begin{array}{c}\text { Crepis foetida } \\
\text { subsp. rhoeadifolia (M.Bieb.) Čelak }\end{array}$ & Prodanović, 2007 & stinking hawksbeard \\
\hline Crepis sancta (L.) Bornm. & Pavlović, 1967; Prodanović, 2007 & holy hawksbeard \\
\hline Crepis setosa Haller f. & Prodanović, 2007 & bristly hawksbeard \\
\hline Crupina vulgaris Pers ex Cass. & $\begin{array}{l}\text { Ranđelović et al., 1982; Prodanović, } \\
2007 \\
\end{array}$ & bearded creeper \\
\hline Cyanus segetum Hill & Prodanović, 2007 & cornflower, bachelor's button \\
\hline $\begin{array}{c}\text { Cyanus triumfettii (All.) Dostál ex Á. } \\
\text { Löve \& D.Löve }\end{array}$ & Prodanović, 2007 & mountain cornflower \\
\hline $\begin{array}{l}\text { Cyanus triumfettii (All.) Dostál ex Á. } \\
\text { Löve \& D.Löve } \\
\text { subsp. axillaries (Čelak.) Štepánek }\end{array}$ & Prodanović, 2007 & mountain cornflower \\
\hline Doronicum columnae Ten. & $\begin{array}{c}\text { Prodanović, (unpublished, field } \\
\text { observation) }\end{array}$ & leopard's bane \\
\hline Doronicum hungaricum (Sadler) Rchb.f & \begin{tabular}{|c|} 
Prodanović, 2007; Krivošej \& \\
Prodanović, 2011; Prodanović et al.,2012
\end{tabular} & \\
\hline Doronicum orientale Hoffm. & $\begin{array}{c}\begin{array}{c}\text { Prodanović, (unpublished, field } \\
\text { observation) }\end{array} \\
\end{array}$ & leopard's bane \\
\hline $\begin{array}{c}\text { Echinops ritro } \\
\text { subsp. ruthenicus (M.Bieb.) Nyman }\end{array}$ & Prodanović, 2007 & southern globethistle \\
\hline Echinops sphaerocephalus L. & Prodanović, 2007 & glandular globe-thistle \\
\hline Erigeron acer $\mathrm{L}$. & Prodanović, 2007 & bitter fleabane \\
\hline Erigeron canadensis L. & Prodanović, 2007 & horseweed, canadian horseweed \\
\hline Eupatorium cannabinum L. & Prodanović, 2007 & hemp-agrimony, holy rope \\
\hline Filago arvensis L. & Prodanović, 2007 & field cottonrose \\
\hline Gallatella albanica Degen & $\begin{array}{l}\text { Rexhepi 1992; Krivošej et al., 1993; } \\
\text { Prodanović, 2007; Prodanović et al., } \\
2008\end{array}$ & \\
\hline Gallatela linosyris (L.) Rchb. & Prodanović, 2007 & goldilocks aster \\
\hline $\begin{array}{l}\text { Hieracium bauhini Besser } \\
\text { subsp. besserianum Spreng }\end{array}$ & Prodanović, 2007 & hawkweed \\
\hline $\begin{array}{c}\text { Hieracium bauhini Besser } \\
\text { subsp. filiferum (Tausch) Zahn }\end{array}$ & Prodanović, 2007 & hawkweed \\
\hline
\end{tabular}




\begin{tabular}{|c|c|c|}
\hline CLASS/ FAMILY/Species & Source/Literature & Common English names \\
\hline $\begin{array}{c}\text { Hieracium bauhini Besser } \\
\text { subsp. heathinum (N.P.) Zahn }\end{array}$ & Prodanović, 2007 & hawkweed \\
\hline $\begin{array}{l}\text { Hieracium bauhini Besser } \\
\text { subsp. pseudo-kerneri Zahn }\end{array}$ & Prodanović, 2007 & hawkweed \\
\hline $\begin{array}{c}\text { Hieracium bauhini Besser } \\
\text { subsp. pseudosparsum Zahn }\end{array}$ & Prodanović, 2007 & hawkweed \\
\hline Hieracium cymosum Vill. & Prodanović, 2007 & hawkweed \\
\hline Hieracium hoppeanum Wallr. ex Nyman & Prodanović, 2007 & Hoppe's hawkweed \\
\hline $\begin{array}{c}\text { Hieracium lachenalii Suter } \\
\text { subsp. festinum (Boreau) Zahn. }\end{array}$ & Prodanović, 2007 & $\begin{array}{c}\text { common hawkweed, yellow } \\
\text { hawkweed }\end{array}$ \\
\hline $\begin{array}{c}\text { Hieracium murorum L. } \\
\text { subsp. gentile (Boreau) Sudre }\end{array}$ & Prodanović, 2007 & wall hawkweed \\
\hline $\begin{array}{l}\text { Hieracium schmidtii } \\
\text { subsp.pallidum (Biv.) O.Bolòs \& Vigo }\end{array}$ & Prodanović, 2007 & Schmidt's hawkweed \\
\hline $\begin{array}{l}\text { Hieracium sabaudum } \mathrm{L} . \\
\text { subsp. vagum Zahn }\end{array}$ & Prodanović, 2007 & $\begin{array}{c}\text { new england hawkweed, } \\
\text { european hawkweed }\end{array}$ \\
\hline Hypochaeris maculata $\mathrm{L}$. & Prodanović, 2007 & spotted cat's ear \\
\hline Hypochaeris radicata $\mathrm{L}$. & Prodanović, 2007 & $\begin{array}{l}\text { catsear, hairy cat's ear, false } \\
\text { dandelion }\end{array}$ \\
\hline Inula britannica $\mathrm{L}$. & Prodanović, 2007 & $\begin{array}{l}\text { british elecampane, british } \\
\text { yellowhead }\end{array}$ \\
\hline Inula conyza (Griess.) DC. & Prodanović, 2007 & ploughman's spikenard \\
\hline Inula ensifolia $\mathrm{L}$. & Rexhepi, 1979; Prodanović, 2007 & swordleaf inula \\
\hline Inula hirta $\mathrm{L}$. & Prodanović, 2007 & hairiness inula \\
\hline Inula oculus-christi $\mathrm{L}$. & Prodanović, 2007 & Christ's eye hoary fleabane \\
\hline Inula salicina $\mathrm{L}$. & Prodanović, 2007 & irish fleabane \\
\hline Jurinea mollis (L.) Rchb. & Rexhepi, 1979; Prodanović, 2007 & \\
\hline $\begin{array}{l}\text { Klasea radiata (Waldst. \& Kit.) A.Löve } \\
\text { \& D. Löve }\end{array}$ & Prodanović, 2007 & radiating outwords \\
\hline Lactuca muralis (L.) Gaertn. & Prodanović, 2007 & wall lettuce \\
\hline Lactuca perennis L. & Prodanović, 2007 & mountain lettuce, blue lettuce \\
\hline Lactuca serriola $\mathrm{L}$. & Prodanović, 2007 & prickly lettuce \\
\hline Lactuca viminea (L.) J. Presl.\& C. Presl. & Prodanović, 2007 & pliant lettuce \\
\hline Leontodon hispidus L. & Prodanović, 2007 & bristly hawkbit \\
\hline Leucanthemum vulgare (Vaill) Lam. & Prodanović, 2007 & ox-eye daisy \\
\hline Matricaria chamomilla $\mathrm{L}$. & Prodanović, 2007 & chamomile \\
\hline Onopordum acanthium L. & Prodanović, 2007 & cotton thistle \\
\hline Petasites hybridus (L.) G.Gaertn. \&al. & Prodanović, 2007 & butterbur \\
\hline Pilosella bauhini (Schult.) Arv.-Touv. & Prodanović, 2007 & \\
\hline Pilosella piloselloides (Vill.) Soják & Prodanović, 2007 & hawkweed \\
\hline Podospermum laciniatum (L.) DC. & Prodanović, 2007 & divided -eaved viper's grass \\
\hline Pulicaria dysenterica (L.) Gaertn. & Prodanović, 2007 & common fleabane \\
\hline Scolymus hispanicus L. & Krasniqi et al., 1981 & $\begin{array}{l}\text { golden thistle, spanish oyster } \\
\text { thistle }\end{array}$ \\
\hline $\begin{array}{l}\text { Scorzonera austriaca Willd. } \\
\text { f. latifolia } \text { Vis. }\end{array}$ & $\begin{array}{l}\text { Pavlović, 1967; Rexhepi, 1979; } \\
\text { Prodanović, } 2007\end{array}$ & \\
\hline $\begin{array}{c}\text { Scorzonera hispanica } \mathrm{L} . \\
\text { var. glastifolia (Willd.) Wallr. }\end{array}$ & Prodanović, 2007 & black salsify, spanish salsify \\
\hline Senecio erucifolius L. & Prodanović, 2007 & hoary ragwort \\
\hline $\begin{array}{c}\text { Senecio leucanthemifolius } \\
\text { subsp. vernalis (Waldst. \& Kit.) Greuter }\end{array}$ & Prodanović, 2007 & eastern groundsel \\
\hline Serratula tinctoria $\mathrm{L}$ & Prodanović, 2007 & \begin{tabular}{|c}
$\begin{array}{c}\text { dyer's plumeless saw-wort, saw- } \\
\text { wort }\end{array}$ \\
\end{tabular} \\
\hline Solidago virgaurea $\mathrm{L}$. & Prodanović, 2007 & goldenrod, woundwort \\
\hline $\begin{array}{c}\text { Sonchus asper (L.) Hill } \\
\text { subsp. glaucescens (Jord.) Ball ex Ball }\end{array}$ & Prodanović, 2007 & prickly sow-thistle \\
\hline Tanacetum corymbosum (L.) Sch.-Bip. & Prodanović, 2007 & corymbflower tansy \\
\hline Tanacetum vulgare $\mathrm{L}$. & Prodanović, 2007 & common tansy, garden tansy \\
\hline Taraxacum campyloides G.E.Haglund & Prodanović, 2007 & dandelion \\
\hline
\end{tabular}




$$
-7302-
$$

\begin{tabular}{|c|c|c|}
\hline CLASS/ FAMILY/Species & Source/Literature & Common English names \\
\hline Tephroseris papposa (Rchb.) Schur. & Prodanović, 2007 & \\
\hline Tragopogon balcanicus Velen. & Prodanović, 2007 & \\
\hline $\begin{array}{l}\text { Tragopogon dubius Scop. } \\
\text { subsp. major (Jacq.) Vollm }\end{array}$ & Prodanović, 2007 & yellow salsify \\
\hline Tragopogon dubius Scop. & $\begin{array}{c}\text { Prodanović et al., 2004; Prodanović, } \\
2007 \\
\end{array}$ & yellow salsify \\
\hline $\begin{array}{c}\text { Tragopogon pratensis } \\
\text { subsp. orientalis (L.) Čelak. }\end{array}$ & Prodanović, 2007 & $\begin{array}{c}\text { ack-go-to-bed-at-noon, meadow } \\
\text { salsify }\end{array}$ \\
\hline Tragopogon pterodes Petrović & Pavlović, 1967; & \\
\hline Tussilago farfara $\mathrm{L}$. & Prodanović, 2007; Krivošej et al., 2013 & coltsfoot \\
\hline $\begin{array}{c}\text { Xanthium orientale } \\
\text { subsp. italicum (Moretti) Greuter }\end{array}$ & Prodanović, 2007 & rough cocklebur, clotbur \\
\hline Xeranthemum аппиит $\mathrm{L}$. & $\begin{array}{c}\text { Pavlović, 1967; Rexhepi, 1979; } \\
\text { Ranđelović et al., 1982; Prodanović, } \\
2007\end{array}$ & annual everlasting, immortelle \\
\hline \multicolumn{3}{|l|}{ CONVOLVULACEAE } \\
\hline Calystegia sepium (L.) R.Br. & Prodanović, 2007 & hedge bindweed \\
\hline Convolvulus arvensis $\mathrm{L}$. & Prodanović, 2007 & field bindweed \\
\hline Convolvulus cantabrica $\mathrm{L}$. & $\begin{array}{c}\text { Pavlović, 1967; Rexhepi, 1979; } \\
\text { Ranđelović et al., 1982; Prodanović et } \\
\text { al., 2004; Prodanović, } 2007\end{array}$ & cantabrican morning glory \\
\hline Cuscuta epithymum (L.) L. & Prodanović, 2007 & dodder, lesser dodder \\
\hline Cuscuta europaea L. & Prodanović, 2007 & greater dodder, european dodder \\
\hline \multicolumn{3}{|l|}{ CORNACEAE } \\
\hline Cornus mas L. & Prodanović, 2007 & cornelian cherry dogwood \\
\hline Cornus sanguinea L. & Prodanović, 2007 & common dogwood \\
\hline \multicolumn{3}{|l|}{ CRASSULACEAE } \\
\hline Hylotelephium telephium (L.) H. Ohba. & Prodanović, 2007 & orpine, livelong, life-everlasting \\
\hline Sedum acre $\mathrm{L}$. & $\begin{array}{c}\text { Rexhepi, 1979; Ranđelović et al., 1982; } \\
\text { Prodanović et al., 2004; Prodanović, } \\
2007\end{array}$ & goldmoss stonecrop \\
\hline Sedum album $\mathrm{L}$. & $\begin{array}{c}\text { Pavlović, 1967; Rexhepi, 1979; } \\
\text { Prodanović et al., 2004; Prodanović, } \\
2007\end{array}$ & white stonecrop \\
\hline Sedum dasyphyllum L. & Rexhepi, 1979; Prodanović, 2007 & corsican stonecrop \\
\hline Sedum hispanicum L. & Prodanović, 2007 & spanish stonecrop \\
\hline Sedum ochroleucum Chaix & Prodanović, 2007 & european stonecrop \\
\hline Sedum sexangulare L. & Prodanović, 2007 & tasteless stonecrop \\
\hline Sempervivum heuffelii Schott & Pavlović, 1967; Prodanović, 2007 & houseleeks \\
\hline Sempervivum marmoreum Griseb. & Prodanović, 2007 & house leek (houseleek) \\
\hline \multicolumn{3}{|l|}{ CUCURBITACEAE } \\
\hline $\begin{array}{c}\text { Bryonia cretica } \\
\text { subsp. dioica (Jacq.) Tutin }\end{array}$ & Prodanović, 2007 & english mandrake \\
\hline $\begin{array}{c}\text { Echinocystis lobata (Michx) Torr. \& A. } \\
\text { Gray }\end{array}$ & Prodanović, 2007 & $\begin{array}{c}\text { wild cucumber, prickly } \\
\text { cucumber }\end{array}$ \\
\hline \multicolumn{3}{|l|}{ CYPERACEAE } \\
\hline Carex acuta $\mathrm{L}$. & Prodanović, 2007 & acute sedge \\
\hline Carex appropinquata Schumach. & Prodanović, 2007 & fibrous tussock-sedge \\
\hline Carex caryophyllea Latour. & Prodanović, 2007 & vernal sedge \\
\hline Carex distans $\mathrm{L}$. & Prodanović, 2007 & distant sedge \\
\hline Carex divulsa Stokes. & Prodanović, 2007 & grey sedge \\
\hline Carex filiformis $\mathrm{L}$. & Prodanović, 2007 & downy-fruited sedge \\
\hline Carex hirta $\mathrm{L}$. & Prodanović, 2007 & hairy sedge \\
\hline Carex leporina $\mathrm{L}$. & Prodanović, 2007 & eggbract sedge, oval sedge \\
\hline Carex montana $\mathrm{L}$. & Prodanović, 2007 & mountain, soft-leaved sedge \\
\hline $\begin{array}{c}\text { Carex muricata } \\
\text { subsp. pairae (F.W.Schultz.) Čelak. }\end{array}$ & Prodanović, 2007 & rough sedge, prickly sedge \\
\hline Carex ornithopoda Willd. & Prodanović et al., 2004; Prodanović, & bird-foot sedge \\
\hline
\end{tabular}




$$
\text { - } 7303 \text { - }
$$

\begin{tabular}{|c|c|c|}
\hline CLASS/ FAMILY/Species & Source/Literature & Common English names \\
\hline & 2007 & \\
\hline Carex panicea $\mathrm{L}$. & Prodanović, 2007 & carnation sedge \\
\hline Carex pendula Huds. & Prodanović, 2007 & pendulous sedge, hanging sedge \\
\hline Carex riparia Curtis & Prodanović, 2007 & greater pond sedge \\
\hline Carex spicata Huds. & Prodanović, 2007 & spiked sedge \\
\hline Carex vulpina $\mathrm{L}$. & Prodanović, 2007 & true fox sedge \\
\hline Schoenoplectus lacustris (L.) Palla & Prodanović, 2007 & lakeshore bulrush \\
\hline \multicolumn{3}{|l|}{ DIPSACACEAE } \\
\hline Lomelosia palaestina (L.) Raf. & Prodanović et al.,2010 & palestine scabious \\
\hline \multicolumn{3}{|l|}{ EUPHORBIACEAE } \\
\hline Euphorbia amygdaloides L. & Prodanović, 2007; Krivošej et al., 2013 & wood spurge \\
\hline Euphorbia barrelieri Savi & Prodanović, 2007 & spurge \\
\hline Euphorbia cyparissias L. & $\begin{array}{l}\text { Prodanović et al., 2004; Prodanović, } \\
2007\end{array}$ & cypress spurge \\
\hline $\begin{array}{c}\text { Euphorbia esula } \\
\text { subsp. tommasniana (Bertol.) Kuzmanov }\end{array}$ & Prodanović, 2007 & green spurge, leafy spurge \\
\hline Euphorbia falcata $\mathrm{L}$. & Prodanović, 2007 & sickle spurge \\
\hline Euphorbia glabriflora Vis. & $\begin{array}{c}\text { Pavlović, 1967; Rexhepi, 1979; } \\
\text { Ranđelović et al., 1982; Krivošej et al., } \\
\text { 1993; Prodanović, } 2007 \\
\end{array}$ & spurge \\
\hline Euphorbia glareosa Pall. ex M.Bieb. & Prodanović, 2007 & spurge \\
\hline Euphorbia helioscopia L. & Prodanović, 2007 & sun spurge \\
\hline Euphorbia ilirica Lam. & Prodanović, 2007 & illyrian spurge \\
\hline Euphorbia myrsinites L. & $\begin{array}{c}\text { Rexhepi, 1979; Ranđelović et al., 1982; } \\
\text { Prodanović, } 2007\end{array}$ & myrtle spurge, blue spurge \\
\hline Euphorbia platyphyllos L. & Prodanović, 2007 & broadleaved spurge \\
\hline Euphorbia salicifolia Host. & Prodanović, 2007 & spurge \\
\hline Euphorbia stricta L. & Prodanović, 2007 & tintern spurge \\
\hline Euphorbia taurinensis All. & Prodanović, 2007 & spurge \\
\hline Mercurialis ovata Sternb. \& Hoppe & Prodanović, 2007 & \\
\hline Mercurialis perennis $\mathrm{L}$. & Prodanović, 2007 & dog's mercury \\
\hline \multicolumn{3}{|l|}{ FAGACEAE } \\
\hline Quercus cerris L. & $\begin{array}{c}\text { Prodanović, et al., 2004; Prodanović, } \\
2007\end{array}$ & turkey oak \\
\hline Quercus frainetto Ten. & $\begin{array}{c}\text { Prodanović et al., 2004; Prodanović, } \\
2007\end{array}$ & hungarian oak \\
\hline Quercus petraea (Matt.) Liebl. & Prodanović, 2007 & sessile oak \\
\hline Quercus pubescens Willd. & $\begin{array}{c}\text { Pavlović, 1967; Prodanović et al., 2004; } \\
\text { Prodanović, } 2007\end{array}$ & downy oak, pubescent oak \\
\hline \multicolumn{3}{|l|}{ GENTIANACEAE } \\
\hline Centaurium erythraea Rafn & Prodanović, 2007 & european centaury \\
\hline Gentiana cruciata $\mathrm{L}$. & Prodanović, 2007 & cross gentian \\
\hline \multicolumn{3}{|l|}{ GERANIACEAE } \\
\hline Erodium ciconium (L.) L' Hér. & Prodanović, 2007 & $\begin{array}{c}\begin{array}{c}\text { redstem filaree, redstem stork's } \\
\text { bill }\end{array} \\
\end{array}$ \\
\hline Erodium cicutarium (L.) L'Hér. & Prodanović, 2007 & $\begin{array}{c}\text { redstem filaree, redstem stork's } \\
\text { bill }\end{array}$ \\
\hline Geranium columbinum $\mathrm{L}$. & Prodanović, 2007 & $\begin{array}{l}\text { long-stalked crane's-bill, } \\
\text { longstalk cranesbill }\end{array}$ \\
\hline Geranium dissectum Jusl. & Prodanović, 2007 & cut-leaved crane's-bill \\
\hline Geranium lucidum L. & Prodanović, 2007 & $\begin{array}{l}\text { shining cranesbill, shiny } \\
\text { geranium }\end{array}$ \\
\hline Geranium molle L. & Prodanović, 2007 & dove's-foot crane's-bill \\
\hline Geranium phaeum L. & Prodanović, 2007 & dusky crane's-bill \\
\hline Geranium pyrenaicum Burm.f. & Prodanović, 2007 & hedgerow cranesbill \\
\hline Geranium robertianum $\mathrm{L}$. & Prodanović, 2007 & herb-Robert, red robin \\
\hline Geranium sanguineum $\mathrm{L}$. & Prodanović, 2007 & bloody crane's-bill \\
\hline
\end{tabular}




$$
\text { - } 7304 \text { - }
$$

\begin{tabular}{|c|c|c|}
\hline CLASS/ FAMILY/Species & Source/Literature & Common English names \\
\hline Crocus chrysanthus (Herb.) Herb. & Prodanović, 2007 & golden crocus \\
\hline Iris graminea $\mathrm{L}$. & Prodanović, 2007; Krivošej et al., 2013 & grass leaved Iris \\
\hline Iris pseudacorus L. & Prodanović, 2007 & yellow iris, water flag \\
\hline Iris reichenbachii Heuff. & Prodanović, 2007 & \\
\hline \multicolumn{3}{|l|}{ JUNCACEAE } \\
\hline Juncus bufonius L. & Prodanović, 2007 & toad rush \\
\hline Juncus compressus Jacq. & Prodanović, 2007 & compressed rush \\
\hline Juncus inflexus $\mathrm{L}$. & Prodanović, 2007 & hard rush, blue rush \\
\hline Luzula campestris (L.) DC. & Prodanović, 2007 & field wood-rush \\
\hline Luzula forsteri (Sm.) DC. & Prodanović, 2007 & southern wood-rush \\
\hline \multicolumn{3}{|l|}{ LAMIACEAE } \\
\hline Ajuga chamaepitys (L.) Schreb. & $\begin{array}{l}\text { Prodanović et al., 2004; Prodanović, } \\
2007\end{array}$ & yellow bugle, ground-pine \\
\hline $\begin{array}{c}\text { Ajuga chamaepitys } \\
\text { subsp.chia (Schreb.) Arcang. }\end{array}$ & Prodanović, 2007 & yellow bugle, ground-pine \\
\hline Ajuga genevensis $\mathrm{L}$. & Prodanović, 2007; Krivošej et al., 2013 & blue bugle, geneva bugleweed \\
\hline Ajuga laxmannii (Murray) Benth. & Prodanović, 2007 & bugleweed, ground pine \\
\hline Ballota nigra $\mathrm{L}$. & Prodanović, 2007 & black horehound \\
\hline Clinopodium acinos (L.) Kuntze & $\begin{array}{l}\text { Prodanović et al., 2004; Prodanović, } \\
2007\end{array}$ & basil thyme, spring savory \\
\hline Clinopodiuma alpinum (L.) Kuntze & Prodanović, 2007 & alpine calamint \\
\hline $\begin{array}{c}\text { Clinopodium nepeta } \\
\text { subsp. glandulosum (Req.) Govaerts }\end{array}$ & Prodanović, 2007 & lesser calamint \\
\hline Clinopodium thymifolium (Scop.) Kuntze & Prodanović, 2007 & $\begin{array}{l}\text { clinopodium thymifolium from } \\
\text { the wild }\end{array}$ \\
\hline Clinopodium vulgare L. & Prodanović, 2007 & wild basil \\
\hline Galeopsis speciosa Mill. & Prodanović, 2007 & arge-flowered hempnettle \\
\hline Glechoma hederacea L. & Prodanović, 2007 & creeping charlie \\
\hline Glechoma hirsuta Waldst.\& Kit. & Prodanović, 2007 & ground-ivy \\
\hline Lamium amplexicaule $\mathrm{L}$. & Prodanović, 2007 & common henbit \\
\hline $\begin{array}{c}\text { Lamium bifidum } \\
\text { subsp. balcanicum Velen. }\end{array}$ & Prodanović, 2007 & dead-nettles \\
\hline Lamium galeobdolon (L.) L. & Prodanović, 2007 & $\begin{array}{l}\text { yellow archangel, yellow } \\
\text { deadnettle }\end{array}$ \\
\hline $\begin{array}{l}\text { Lamium garganicum } \mathrm{L} . \\
\text { subsp. garganicum }\end{array}$ & Prodanović, 2007 & large dead nettle \\
\hline $\begin{array}{c}\text { Lamium garganicum L. } \\
\text { subsp. glabratum (Griseb.) Briq. }\end{array}$ & Prodanović, 2007 & large dead nettle \\
\hline Lamium purpureum $\mathrm{L}$. & Prodanović, 2007 & red dead-nettle \\
\hline Leonurus cardiaca $\mathrm{L}$. & Prodanović, 2007 & motherwort in english \\
\hline Lycopus europaeus L. & Prodanović, 2007 & gypsywort \\
\hline Marrubium peregrinum $\mathrm{L}$. & Prodanović, 2007 & horehound \\
\hline Marrubium vulgare $\mathrm{L}$. & Prodanović, 2007 & $\begin{array}{c}\text { white horehound, common } \\
\text { horehound }\end{array}$ \\
\hline Melittis melissophyllum L. & Prodanović, 2007 & bastard balm \\
\hline Mentha aquatica $\mathrm{L}$. & Prodanović, 2007 & water mint \\
\hline Mentha longifolia (L.) L. & Prodanović, 2007; Krivošej et al., 2013 & asian mint \\
\hline Nepeta cataria L. & Prodanović, 2007 & catnip, catswort \\
\hline Origanum vulgare $\mathrm{L}$. & Prodanović, 2007 & origanum, wild marjoram \\
\hline Phlomis tuberosa $\mathrm{L}$. & Prodanović et al.,2018 & tuberous jerusalem sage \\
\hline Prunella laciniata (L.) L. & $\begin{array}{c}\text { Ranđelović et al., 1982; Prodanović, } \\
2007\end{array}$ & cutleaf selfheal \\
\hline Prunella vulgaris $\mathrm{L}$. & Prodanović, 2007 & common self-heal, heal-all \\
\hline Salvia amplexicaulis Lam. & Prodanović, 2007 & stem-clasping sage \\
\hline Salvia nemorosa L. & Prodanović, 2007 & woodland sage, balkan clary \\
\hline $\begin{array}{c}\text { Salvia pratensis L. } \\
\text { subsp. pozegensis (Watzl) Diklic }\end{array}$ & $\begin{array}{c}\text { Prodanović, 2007; Prodanović et al., } \\
2008 \\
\end{array}$ & meadow sage \\
\hline Salvia sclarea $\mathrm{L}$. & Prodanović et al., 2004; Prodanović, & salvia romana, clary sage \\
\hline
\end{tabular}




$$
-7305 \text { - }
$$

\begin{tabular}{|c|c|c|}
\hline CLASS/ FAMILY/Species & Source/Literature & Common English names \\
\hline & 2007 & \\
\hline Salvia verticillata $\mathrm{L}$. & Prodanović, 2007 & lilac sage \\
\hline Scutellaria altissima $\mathrm{L}$. & Prodanović, 2007 & tall skullcap \\
\hline Scutellaria galericulata $\mathrm{L}$. & Prodanović, 2007 & marsh skullcap, hooded skullcap \\
\hline Sideritis montana $\mathrm{L}$. & $\begin{array}{l}\text { Prodanović et al., 2004; Prodanović, } \\
2007\end{array}$ & mountain ironwor \\
\hline Stachys germanica $\mathrm{L}$. & Prodanović, 2007 & downy woundwort \\
\hline Stachys officinalis (L.) Trevis & Prodanović, 2007 & common hedgenettle, betony \\
\hline Stachys palustris L. & Prodanović, 2007 & marsh woundwort \\
\hline $\begin{array}{c}\text { Stachys recta } \\
\text { subsp. baldacii (K. Malý) Hayek }\end{array}$ & $\begin{array}{c}\text { Ranđelović et al., 1982; Rexhepi, 1979; } \\
\text { Prodanović, } 2007\end{array}$ & stiff hedgenettle \\
\hline $\begin{array}{c}\text { Stachys recta } \mathrm{L} . \\
\text { subsp. recta } \\
\text { var. chrysophaea (Pančić) Hayek } \\
\text { f. chrysophae }\end{array}$ & Prodanović, 2007 & stiff hedgenettle \\
\hline Stachys scardica (Gris.) Hayek & Rexhepi, 1979; Prodanović, 2007 & \\
\hline Teucrium chamaedrys $\mathrm{L}$. & $\begin{array}{l}\text { Prodanović et al., 2004; Prodanović, } \\
2007\end{array}$ & wall germander \\
\hline Teucrium montanum L. & $\begin{array}{c}\text { Pavlović, 1967; Ranđelović et al, 1982; } \\
\text { Prodanović, } 2007\end{array}$ & mountain germander \\
\hline $\begin{array}{l}\text { Teucrium montanum } \mathrm{L} . \\
\text { subsp. montanum }\end{array}$ & Prodanović, 2007 & mountain germander \\
\hline Thymus longicaulis C. Presl. & Pavlović, 1967 & thyme \\
\hline $\begin{array}{c}\text { Thymus pulegioides } \\
\text { subsp. montanus (Benth.) Ronniger. }\end{array}$ & Prodanović, 2007 & broad-leaved thyme \\
\hline Ziziphora capitata $\mathrm{L}$ & Prodanović, 2007 & oriental zizifora \\
\hline \multicolumn{3}{|l|}{ LEGUMINOSAE } \\
\hline Amorpha fruticosa $\mathrm{L}$. & Prodanović, 2007 & false indigo bush \\
\hline Astragalus cicer $\mathrm{L}$. & Prodanović, 2007 & chickpea milkvetch \\
\hline Astragalus dasyanthus Pall. & $\begin{array}{l}\text { Prodanović, 2007; Prodanović et al., } \\
\text { 2008; Prodanović et al.,2012 }\end{array}$ & milkvetch \\
\hline Astragalus glycyphyllos L. & Prodanović, 2007 & liquorice milkvetch \\
\hline Astragalus hamosus L. & Prodanović, 2007 & southern milk vetch \\
\hline Astragalus onobrychis L. & $\begin{array}{c}\text { Pavlović, 1967; Ranđelović et al., 1982; } \\
\text { Prodanović, } 2007\end{array}$ & sainfoin milk vetch \\
\hline $\begin{array}{l}\text { Chamaecytisus ciliatus } \\
\text { var. alpestris (Schur.) Diklić }\end{array}$ & Prodanović, 2007 & \\
\hline Colutea arborescens $\mathrm{L}$. & Prodanović, 2007 & bladder senna \\
\hline Coronilla scorpioides (L.) Koch. & Prodanović, 2007 & $\begin{array}{l}\text { yellow crown vetch, annual } \\
\text { scorpion-vetch }\end{array}$ \\
\hline $\begin{array}{c}\text { Cytisus austriacus } \\
\text { subsp. heuffelii (Wierzb.) Asch. \& Graebn }\end{array}$ & Prodanović, 2007 & broom \\
\hline Cytisus decumbens (Durande) Spach. & $\begin{array}{l}\text { Ranđelović et al., 1982; Prodanović, } \\
2007\end{array}$ & scotch broom \\
\hline Cytisus hirsutus L. & Prodanović, 2007 & clustered broom \\
\hline Cytisus procumbens (Willd.) Spreng. & Prodanović, 2007 & broom \\
\hline $\begin{array}{c}\text { Dorycnium pentaphyllum } \\
\text { subsp. germanicum (Gremli) Gams }\end{array}$ & $\begin{array}{l}\text { Pavlović, 1967; Rexhepi, 1979; } \\
\text { Prodanović, } 2007\end{array}$ & prostrate canary clover, badassi \\
\hline $\begin{array}{c}\text { Dorycnium pentaphyllum } \\
\text { subsp. herbaceum (Vill.) Rouy }\end{array}$ & $\begin{array}{c}\text { Prodanović et al., 2004; Prodanović, } \\
2007 \\
\end{array}$ & prostrate canary clover, badassi \\
\hline Genista januensis Viv. & Prodanović, 2007 & broom \\
\hline Genista sagittalis $\mathrm{L}$. & Prodanović, 2007; Krivošej et al., 2013 & winged broom \\
\hline Genista tinctoria $\mathrm{L}$. & Prodanović, 2007 & dyer's greenweed, dyer's broom \\
\hline Hippocrepis comosa L. & Prodanović, 2007 & horseshoe vetch \\
\hline Hippocrepis emerus (L.) Lassen & $\begin{array}{l}\text { Ranđelović et al., 1982; Prodanović, } \\
2007\end{array}$ & scorpion senna \\
\hline Lathyrus hallersteinii Baumg. & Prodanović, 2007 & yellow pea \\
\hline Lathyrus hirsutus L. & Prodanović, 2007 & $\begin{array}{l}\text { caley pea, singletary pea, hairy } \\
\text { vetchling }\end{array}$ \\
\hline
\end{tabular}




$$
\text { - } 7306 \text { - }
$$

\begin{tabular}{|c|c|c|}
\hline CLASS/ FAMILY/Species & Source/Literature & Common English names \\
\hline Lathyrus latifolius L. & Prodanović, 2007 & everlasting-pea, perennial pea \\
\hline Lathyrus niger (L.) Bernh. & Prodanović, 2007 & black pea \\
\hline Lathyrus nissolia L. & Prodanović, 2007 & grass vetchling, grass pea \\
\hline Lathyrus pannonicus (Jacq.) Garcke & Prodanović, 2007 & felted vetch \\
\hline Lathyrus pratensis L. & Prodanović, 2007; Krivošej et al., 2013 & meadow vetchling \\
\hline Lathyrus setifolius L. & Prodanović, 2007 & narrow-leaved red vetchling \\
\hline Lathyrus sphaericus Retz. & Prodanović, 2007 & $\begin{array}{l}\text { grass pea, round-seeded } \\
\text { vetchling }\end{array}$ \\
\hline Lathyrus tuberosus L. & Prodanović, 2007 & tuberous pea, tuberous vetchling \\
\hline Lathyrus venetus (Mill.) Wohlf. & $\begin{array}{c}\text { Prodanović et al., 2004; Prodanović, } \\
2007\end{array}$ & bushy vetchling \\
\hline Lathyrus vernus (L.) Bernh. & Prodanović, 2007 & spring vetchling, spring pea \\
\hline Lembotropis nigricans (L.) Griseb. & $\begin{array}{c}\text { Ranđelović et al., 1982; Prodanović, } \\
2007 \\
\end{array}$ & black broom \\
\hline Lens nigricans (M. Bieb.) Godr. & Prodanović, 2007 & lens \\
\hline Lotus corniculatus L. & Prodanović, 2007 & birdsfoot trefoil \\
\hline Medicago arabica (L.) Huds. & Prodanović, 2007 & spotted medick \\
\hline Medicago falcata $\mathrm{L}$. & $\begin{array}{c}\text { Ranđelović et al., 1982; Prodanović, } \\
2007\end{array}$ & yellow lucerne, sickle alfalfa \\
\hline Medicago lupulina $\mathrm{L}$. & $\begin{array}{c}\text { Prodanović et al., 2004; Prodanović, } \\
2007 \\
\end{array}$ & black medick, nonesuch \\
\hline Medicago minima (L.) L. & Prodanović, 2007 & little burclover \\
\hline Medicago orbicularis (L.) Bartal. & Prodanović, 2007 & blackdisk medick, button clover \\
\hline Medicago prostrata Jacq. & $\begin{array}{l}\text { Pavlović, 1967; Rexhepi, 1979; } \\
\text { Prodanović, } 2007 \\
\end{array}$ & alfalfa, wild \\
\hline Medicago rigidula (L.) All. & Prodanović, 2007 & tifton burclover, rigid medick \\
\hline Medicago sativa $\mathrm{L}$. & Prodanović, 2007 & lucerne \\
\hline Melilotus albus Medik. & Prodanović, 2007 & honey clover, white melilot \\
\hline Melilotus officinalis (L.) Pall. & Prodanović, 2007 & yellow sweet clover \\
\hline Onobrychis alba (Waldst. \& Kit.) Desv. & Prodanović, 2007 & sainfoin \\
\hline Onobrychis viciifolia Scop. & Prodanović, 2007 & common sainfoin \\
\hline $\begin{array}{c}\text { Ononis spinosa } \\
\text { subsp. hircina (Jacq.) Gams }\end{array}$ & Prodanović, 2007 & spiny restharrow \\
\hline Ononis pusilla L. & Prodanović, 2007 & \\
\hline Oxytropis pilosa (L.) DC. & $\begin{array}{c}\text { Prodanović, 2007; Prodanović et } \\
\text { al.,2012 }\end{array}$ & hairy milk vetch \\
\hline $\begin{array}{c}\text { Pisum sativum } \\
\text { subsp. elatius (M.Bieb.) Asch.\& Graebn. }\end{array}$ & Prodanović, 2007 & dun pea \\
\hline Robinia pseudoacacia L. & Prodanović, 2004; Prodanović, 2007 & black locust \\
\hline Securigera elegans (Pancic) Lassen & Prodanović, 2007 & crownvetch \\
\hline Securigera varia (L.) Lassen & $\begin{array}{l}\text { Prodanović et al., 2004; Prodanović, } \\
2007\end{array}$ & purple crownvetch \\
\hline Trifolium angustifolium L. & Prodanović, 2007 & narrowleaf crimson clover \\
\hline Trifolium arvense $\mathrm{L}$. & Prodanović, 2007 & hare's-foot clover \\
\hline Trifolium dalmaticum Vis. & Prodanović, 2007 & dalmatian clover \\
\hline Trifolium fragiferum $\mathrm{L}$. & Prodanović, 2007 & strawberry clover \\
\hline Trifolium glomeratum L. & Krivošej \& Prodanović, 2011 & clustered clover \\
\hline Trifolium hirtum All. & Prodanović, 2007 & rose clover \\
\hline Trifolium incarnatum L. & Prodanović, 2007 & crimson clover, italian clover \\
\hline Trifolium medium $\mathrm{L}$. & Prodanović, 2007 & zigzag clover \\
\hline Trifolium montanum L. & Prodanović, 2007; Krivošej et al., 2013 & mountain clover \\
\hline Trifolium ochroleucon Huds. & Prodanović, 2007 & sulphur clover \\
\hline Trifolium patens Schreb. & Prodanović, 2007; Krivošej et al., 2013 & clover \\
\hline Trifolium pignantii Fauchè \& Chaub. & Prodanović, 2007 & clover \\
\hline Trifolium pratense L. & Prodanović, 2007 & red clover \\
\hline Trifolium repens L. & Prodanović, 2007 & white clover, landino clover \\
\hline Trifolium striatum L. & Prodanović, 2007 & knotted clover \\
\hline
\end{tabular}




$$
\text { - } 7307 \text { - }
$$

\begin{tabular}{|c|c|c|}
\hline CLASS/ FAMILY/Species & Source/Literature & Common English names \\
\hline Trifolium strictum Jusl & $\begin{array}{l}\text { Prodanović, 2007; Krivošej \& } \\
\text { Prodanović, } 2011\end{array}$ & upright clover \\
\hline Trifolium trichopterum Pancic & Pavlović, 1967; Prodanović, 2007 & \\
\hline Trigonella esculenta Willd. & Prodanović, 2007 & fenugreek \\
\hline Trigonella gladiata M. Bieb. & Prodanović et al.,2010 & fenugreek \\
\hline Vicia cracca $\mathrm{L}$. & Prodanović, 2007 & $\begin{array}{l}\text { tufted vetch, cow vetch, } \\
\text { bird vetch }\end{array}$ \\
\hline $\begin{array}{c}\text { Vicia cracca } \\
\text { subsp. incana (Gouan) Rouy. }\end{array}$ & Prodanović, 2007 & $\begin{array}{l}\text { tufted vetch, cow vetch, } \\
\text { bird vetch }\end{array}$ \\
\hline Vicia grandiflora Scop. & Prodanović, 2007 & $\begin{array}{c}\text { large-flowered vetch, large } \\
\text { yellow vetch }\end{array}$ \\
\hline Vicia hirsuta (L.) Gray & Prodanović, 2007 & hairy tare, hairy vetch \\
\hline Vicia lathyroides L. & Prodanović, 2007 & spring vetch \\
\hline Vicia pannonica Crantz & Prodanović, 2007 & hungarian vetch \\
\hline Vicia peregrina $\mathrm{L}$. & Prodanović, 2007 & wandering vetch \\
\hline Vicia pisiformis $\mathrm{L}$. & Prodanović, 2007 & pea vetch \\
\hline Vicia sativa $\mathrm{L}$. & Prodanović, 2007 & common vetch \\
\hline $\begin{array}{c}\text { Vicia sativa } \\
\text { subsp. nigra (L.) Ehrh. }\end{array}$ & Prodanović, 2007 & common vetch, garden vetch \\
\hline Vicia sparsiflora Ten. & Prodanović, 2007 & vetch \\
\hline Vicia tenuifolia Roth. & Prodanović, 2007 & fine-leaved vetch, cow vetch \\
\hline Vicia villosa Roth. & Prodanović, 2007 & winter vetch, hairy vetch \\
\hline \multicolumn{3}{|l|}{ LILIACEAE } \\
\hline Erythronium dens-canis L. & Prodanović, 2007 & dog's tooth violet \\
\hline $\begin{array}{l}\text { Fritillaria montana Hoppe ex W.D.J. } \\
\text { Koch }\end{array}$ & Prodanović, 2007 & missionbells \\
\hline Gagea lutea (L.) Ker Gawl. & Prodanović, 2007 & yellow star-of-bethlehem \\
\hline Gagea pusilla (F.W.Schmidt) Sweet & \begin{tabular}{|c|} 
Krivošej et al., 1995-1998, Prodanović, \\
2007
\end{tabular} & yellow star-of-bethlehem \\
\hline Lilium martagon $\mathrm{L}$. & Prodanović, 2007 & martagon lily, turk's cap lily \\
\hline Tulipa sylvestris L. & Prodanović, 2007 & wild tulip, woodland tulip \\
\hline Tulipa serbica Tatić \& Krivošej & $\begin{array}{l}\text { Tatić \& Krivošej, 1997; Prodanović, } \\
\text { 2007; Prodanović et al., } 2008\end{array}$ & serbian tulip \\
\hline \multicolumn{3}{|l|}{ LINACEAE } \\
\hline Linum bienne Mill. & Prodanović, 2007 & pale, narrowleaf flax \\
\hline Linum austriacum $\mathrm{L}$. & Prodanović, 2007 & austrian flax \\
\hline Linum flavum $\mathrm{L}$. & Prodanović, 2007 & golden flax, yellow flax \\
\hline Linum nervosum Waldst.\& Kit. & Krivošej \& Prodanović, 2011 & \\
\hline Linum tenuifolium $\mathrm{L}$. & Pavlović, 1967; Prodanović, 2007 & narrow-leaved flax \\
\hline \multicolumn{3}{|l|}{ LYTHRACEAE } \\
\hline Lythrum salicaria $\mathrm{L}$. & Prodanović, 2007 & purple loosestrife \\
\hline \multicolumn{3}{|l|}{ MALVACEAE } \\
\hline Althaea hirsuta $\mathrm{L}$. & Prodanović, 2007 & hairy marshmallow \\
\hline Althaea officinalis $\mathrm{L}$. & Prodanović, 2007 & marsh-mallow \\
\hline Lavatera thuringiaca $\mathrm{L}$. & Krasniqi et al., 1981; Prodanović, 2007 & garden tree-mallow \\
\hline Malva sylvestris L. & Prodanović, 2007 & high mallow \\
\hline Tilia cordata Mill. & Prodanović, 2007 & small-leaved lime \\
\hline Tilia tomentosa Moench & Prodanović, 2007 & silver lime \\
\hline \multicolumn{3}{|l|}{ MELANTHIACEAE } \\
\hline Veratrum nigrum $\mathrm{L}$. & Prodanović, 2007 & black false hellebore \\
\hline \multicolumn{3}{|l|}{ NYMPHAEACEAE } \\
\hline Nuphar lutea (L.) Sm. & Prodanović et al.,2010 & yellow pond-lily \\
\hline \multicolumn{3}{|l|}{ OLEACEAE } \\
\hline Fraxinus ornus L. & Prodanović et al.,2004; Prodanović, 2007 & manna ash \\
\hline Ligustrum vulgare $\mathrm{L}$. & \begin{tabular}{|l|} 
Prodanović, 2007 \\
\end{tabular} & wild privet \\
\hline \multicolumn{3}{|l|}{ ONAGRACEAE } \\
\hline Epilobium angustifolium $\mathrm{L}$. & Prodanović, 2007 & fireweed \\
\hline
\end{tabular}




$$
-7308 \text { - }
$$

\begin{tabular}{|c|c|c|}
\hline CLASS/ FAMILY/Species & Source/Literature & Common English names \\
\hline Epilobium hirsutum L. & Prodanović, 2007 & great willowherb \\
\hline Epilobium parviflorum Schreb. & Prodanović, 2007 & hoary willowherb \\
\hline Oenothera biennis L. & Prodanović, 2007 & common evening-primrose \\
\hline \multicolumn{3}{|l|}{ ORCHIDACEAE } \\
\hline $\begin{array}{c}\text { Anacamptis morio (L.) R.M.Bateman, } \\
\text { Pridgeon \& M.W.Chase }\end{array}$ & $\begin{array}{c}\text { Prodanović, 2007; Prodanović et al., } \\
2008\end{array}$ & green-winged orchid \\
\hline $\begin{array}{l}\text { Anacamptis papilionaceae (L.) } \\
\text { R.M.Bateman, Pridgeon \& M.W.Chase }\end{array}$ & $\begin{array}{c}\text { Prodanović, 2007; Prodanović et al., } \\
2008\end{array}$ & butterfly orchid \\
\hline Anacamptis pyramidalis (L.) Rich. & Prodanović, 2007 & pyramidal orchid \\
\hline Cephalanthera damasonium (Mill.) Druce & Prodanović, 2007 & white helleborine \\
\hline Cephalanthera rubra (L.) Rich. & $\begin{array}{c}\text { Prodanović, 2007; Prodanović et al., } \\
2008 \\
\end{array}$ & red helleborine \\
\hline Epipactis helleborine (L.) Crantz. & Prodanović, 2007 & broad-leaved helleborine \\
\hline $\begin{array}{l}\text { Epipactis helleborine (L.) Crantz. } \\
\text { subsp. helleborine }\end{array}$ & Prodanović, 2007 & broad-leaved helleborine \\
\hline Epipactis microphylla (Ehrh.) Sw., & $\begin{array}{c}\text { Prodanović et al., 2004; Prodanović, } \\
\text { 2007; Prodanović et al., 2008; }\end{array}$ & small-leaved helleborine \\
\hline $\begin{array}{l}\text { Himantoglossum caprinum (M. Bieb.) } \\
\text { Spreng. }\end{array}$ & Prodanović et al.,2018 & \\
\hline Limodorum abortivum (L.) Sw. & $\begin{array}{c}\text { Prodanović, 2007; Prodanović et al., } \\
2008\end{array}$ & violet limodore \\
\hline Neottia nidus-avis (L.) Rich. & $\begin{array}{c}\text { Prodanović, 2007; Prodanović et al., } \\
\text { 2008; }\end{array}$ & bird's-nest orchid \\
\hline $\begin{array}{l}\text { Neotinea tridentata (Scop.) } \\
\text { R.M.Bateman, Pridgeon \& M.W.Chase }\end{array}$ & $\begin{array}{c}\text { Prodanović, 2007; Prodanović et al., } \\
\text { 2008; Krivošej et al., } 2013\end{array}$ & three-toothed orchid \\
\hline Ophrys apifera Huds. & Prodanović, 2007 & bee orchid \\
\hline Orchis mascula (L.) L. & $\begin{array}{c}\text { Prodanović, 2007; Prodanović et al., } \\
2008 ;\end{array}$ & $\begin{array}{l}\text { early-purple orchid, early } \\
\text { spring orchis }\end{array}$ \\
\hline Orchis purpurea Huds. & $\begin{array}{c}\text { Prodanović, 2007; Prodanović et al., } \\
\text { 2008; }\end{array}$ & lady orchid \\
\hline Platanthera bifolia (L.) Rich & $\begin{array}{c}\text { Prodanović, 2007; Prodanović et al., } \\
2008\end{array}$ & lesser butterfly-orchid \\
\hline \multicolumn{3}{|l|}{ OROBANCHACEAE } \\
\hline Lathraea squamaria L. & Prodanović, 2007 & common toothwort \\
\hline Melampyrum arvense $\mathrm{L}$. & Prodanović, 2007 & field cow-wheat \\
\hline Melampyrum cristatum L. & Prodanović, 2007 & crested cow-wheat \\
\hline $\begin{array}{l}\text { Melampyrum heracleoticum Boiss. \& } \\
\text { Orph. }\end{array}$ & $\begin{array}{c}\text { Prodanović, 2007; Prodanović et al., } \\
2008\end{array}$ & cow wheat \\
\hline Orobanche alba Stephan ex Willd. & Prodanović, 2007 & scalloped broomrape \\
\hline Orobanche caryophyllacea $\mathrm{Sm}$. & Prodanović, 2007 & clove-scented broomrape \\
\hline Parentucellia latifolia Caruel & Prodanović, 2007 & broadleaf glandweed \\
\hline $\begin{array}{c}\text { Pedicularis comosa } \\
\text { subsp. campestris (Griseb. \& Schenk) Soó }\end{array}$ & Prodanović, 2007 & glandweed \\
\hline Pedicularis comosa $\mathrm{L}$ & Prodanović, 2007 & glandweed \\
\hline Pedicularis friderici-augusti Tomm. & Prodanović, 2007 & Frederick Augustus' lousewort \\
\hline Rhinanthus rumelicus Velen. & Prodanović, 2007 & rattle \\
\hline \multicolumn{3}{|l|}{ PAPAVERACEAE } \\
\hline Chelidonium majus L. & Prodanović, 2007 & reater celandine, nipplewort \\
\hline Corydalis cava (L.) Schweigg. \& Körte & Prodanović, 2007 & hollow root \\
\hline Corydalis solida (L.) Clairv. & Prodanović, 2007 & fumewort \\
\hline Papaver dubium L. & Prodanović, 2007 & long-headed poppy \\
\hline Papaver rhoeas L. & Prodanović, 2007 & common poppy, corn poppy \\
\hline \multicolumn{3}{|l|}{ PLANTAGINACEAE } \\
\hline Digitalis laevigata Waldst. \& Kit. & Prodanović, 2007 & grecian foxglove \\
\hline Digitalis lanata Ehrh. & $\begin{array}{l}\text { Prodanović et al., 2004; Prodanović, } \\
2007\end{array}$ & woolly foxglove \\
\hline Linaria genistifolia (L.) Mill. & Pavlović, 1967; Prodanović, 2007 & toadflax \\
\hline $\begin{array}{l}\text { Linaria genistifolia (L.) Mill. } \\
\text { subsp. genistifolia }\end{array}$ & Prodanović, 2007 & broomleaf toadflax \\
\hline
\end{tabular}




\begin{tabular}{|c|c|c|}
\hline CLASS/ FAMILY/Species & Source/Literature & Common English names \\
\hline Linaria vulgaris Mill. & Prodanović, 2007 & yellow toadflax \\
\hline Plantago argentea Chaix & Rexhepi, 1979; Prodanović, 2007 & silver plantain \\
\hline Plantago lanceolata $\mathrm{L}$. & Prodanović, 2007 & ribwort plantain \\
\hline Plantago major L. & Prodanović, 2007 & $\begin{array}{l}\text { roadleaf plantain, white man's } \\
\text { foot, greater plantain }\end{array}$ \\
\hline $\begin{array}{l}\text { Plantago major } \mathrm{L} . \\
\text { subsp. major }\end{array}$ & Prodanović, 2007 & $\begin{array}{l}\text { roadleaf plantain, white man's } \\
\text { foot, greater plantain }\end{array}$ \\
\hline Plantago media $\mathrm{L}$. & Prodanović, 2007 & hoary plantain \\
\hline Plantago subulata $\mathrm{L}$. & & plantain \\
\hline Veronica anagallis-aquatica $\mathrm{L}$. & Prodanović, 2007 & water speedwell \\
\hline Veronica arvensis $\mathrm{L}$. & Prodanović, 2007 & wall speedwell, corn speedwell \\
\hline Veronica austiaca L. & Prodanović, 2007 & $\begin{array}{c}\text { broadleaf speedwell, large } \\
\text { speedwell }\end{array}$ \\
\hline Veronica beccabunga $\mathrm{L}$. & Prodanović, 2007 & european speedwell, brooklime \\
\hline Veronica chamaedrys L. & Prodanović, 2007 & $\begin{array}{c}\text { germander speedwell, bird's-eye } \\
\text { speedwell }\end{array}$ \\
\hline Veronica hederifolia L. & Prodanović, 2007 & ivy-leaved speedwell \\
\hline Veronica officinalis L. & Prodanović, 2007 & heath speedwell \\
\hline Veronica persica Poir. & Prodanović, 2007 & persian speedwel \\
\hline Veronica polita Fr. & Prodanović, 2007 & grey field-speedwell \\
\hline Veronica praecox All. & Prodanović, 2007 & breckland speedwell \\
\hline Veronica prostrata $\mathrm{L}$. & Prodanović, 2007 & rock speedwell \\
\hline Veronica serpyllifolia L. & Prodanović, 2007 & thyme-leaved speedwell \\
\hline $\begin{array}{l}\text { Veronica serpyllifolia } \mathrm{L} . \\
\text { subsp. serpyllifolia }\end{array}$ & Prodanović, 2007 & thyme-leaved speedwell \\
\hline Veronica spicata $\mathrm{L}$. & $\begin{array}{c}\text { Prodanović, (unpublished, field } \\
\text { observation) }\end{array}$ & spike speedwell \\
\hline \multicolumn{3}{|l|}{ PLUMBAGINACEAE } \\
\hline Goniolimon incanum (L.) Hepper & Prodanović, 2007 & lavender statice, sea lavender \\
\hline Goniolimon collinum (Griseb.) Boiss. & Pavlović, 1967; Prodanović, 2007 & sea lavender \\
\hline Goniolimon tataricum (L.) Boiss. & Prodanović, 2007 & german statice, tatarian statice \\
\hline \multicolumn{3}{|l|}{ POACEAE } \\
\hline Aegilops cylindrica Host & Prodanović, 2007 & jointed goatgrass \\
\hline Aegilops geniculata Roth. & Prodanović, 2007 & goat grass, ovate goatgrass \\
\hline Agropyron cristatum (L.) Gaertn. & Rexhepi, 1979; Prodanović, 2007 & crested wheatgrass \\
\hline Agrostis stolonifera L. & Prodanović, 2007 & creeping bentgrass, fiorin \\
\hline Alopecurus myosuroides Huds. & Prodanović, 2007 & mousetail grass, black grass \\
\hline Alopecurus pratensis L. & Prodanović, 2007; Krivošej et al., 2013 & meadow foxtail \\
\hline Anthoxanthum odoratum L. & Prodanović, 2007; Krivošej et al., 2013 & sweet vernal grass \\
\hline Apera spica-venti (L.) P. Beauv. & Prodanović, 2007 & common windgrass \\
\hline $\begin{array}{c}\text { Arrhenatherum elatius (L.) P. Beauv.ex } \\
\text { J.Presl \& C.Presl }\end{array}$ & Prodanović, 2007 & false oat-grass \\
\hline $\begin{array}{c}\text { Brachypodium silvaticum (Huds.) P. } \\
\text { Beauv. }\end{array}$ & Prodanović, 2007 & slender false-brome \\
\hline Bothriochloa ischaemum (L.) & Prodanović, 2007 & yellow bluestem \\
\hline Briza media L. Keng & Prodanović, 2007; Krivošej et al., 2013 & quaking grass \\
\hline Bromus arvensis $\mathrm{L}$. & Prodanović, 2007 & field brome \\
\hline Bromus commutatus Schrad. & Prodanović, 2007 & meadow brome \\
\hline Bromus erectus Huds. & Rexhepi, 1979; Prodanović, 2007 & erect brome, upright brome \\
\hline Bromus hordeaceus L. & Prodanović, 2007 & soft brome \\
\hline Bromus inermis Leyss. & Prodanović, 2007 & smooth brome \\
\hline Bromus racemosus $\mathrm{L}$. & Prodanović, 2007 & smooth brome \\
\hline Bromus squarrosus L. & Prodanović, 2007 & corn brome \\
\hline Bromus sterilis L. & Rexhepi, 1979; Prodanović, 2007 & poverty brome \\
\hline Bromus tectorum $\mathrm{L}$. & Prodanović, 2007 & drooping brome, cheatgrass \\
\hline Chrysopogon gryllus (L.) Trin & Rexhepi, 1979; Prodanović, 2007 & \\
\hline Cynodon dactylon (L.) Pers. & Prodanović, 2007 & bermuda grass, couch grass \\
\hline
\end{tabular}




$$
\text { - } 7310 \text { - }
$$

\begin{tabular}{|c|c|c|}
\hline CLASS/ FAMILY/Species & Source/Literature & Common English names \\
\hline Cynosurus cristatus L. & Prodanović, 2007; Krivošej et al., 2013 & crested dogstail grass \\
\hline Dactylis glomerata L. & $\begin{array}{l}\text { Prodanović et al., 2004; Prodanović, } \\
\text { 2007; Krivošej et al., } 2013\end{array}$ & cock's-foot, orchard grass \\
\hline Danthonia alpina Vest. & Prodanović, 2007; Krivošej et al., 2013 & heathgrass, wallaby grass \\
\hline Dasypyrum villosum (L.) Borbás & Prodanović, 2007 & mosquitograss \\
\hline Eragrostis minor Host & Prodanović, 2007 & ovegrass, canegrass \\
\hline Elymus hispidus (Opiz) Melderis & Prodanović, 2007 & hairy couch grass \\
\hline Elytrigia repens (L.) Nevski. & Prodanović, 2007 & couch grass \\
\hline Festuca arundinacea Schreb. & Prodanović, 2007 & tall fescue \\
\hline Festuca pratensis Huds. & Prodanović, 2007 & meadow fescue \\
\hline Festuca rubra L. & Prodanović, 2007 & red fescue \\
\hline Festuca valesiaca Schleich. ex Gaudin & Prodanović, 2007 & volga fescue \\
\hline Glyceria fluitans (L.) R.Br. & Prodanović, 2007 & $\begin{array}{c}\text { floating sweet-grass, water } \\
\text { mannagrass }\end{array}$ \\
\hline Glyceria notata Chevall. & Prodanović, 2007 & plicate sweet grass \\
\hline $\begin{array}{c}\text { Helictotrichon compressum (Heuff.) } \\
\text { Henrard }\end{array}$ & Prodanović, 2007 & \\
\hline Holcus lanatus L. & Prodanović, 2007 & yorkshire fog, tufted grass \\
\hline $\begin{array}{c}\text { Hordeum murinum } \\
\text { subsp. leporinum (Link) Arcang. }\end{array}$ & Prodanović, 2007 & wall barley \\
\hline Koeleria macrantha (Ledeb.) Schult. & $\begin{array}{c}\text { Prodanović, (unpublished, field } \\
\text { observation) }\end{array}$ & prairie junegrass \\
\hline Koeleria pyramidata (Lam.) P. Beauv. & Prodanović, 2007 & \\
\hline Lolium perenne L. & Prodanović, 2007 & $\begin{array}{c}\text { perennial ryegrass, english } \\
\text { ryegrass }\end{array}$ \\
\hline Melica ciliata $\mathrm{L}$. & $\begin{array}{c}\text { Pavlović, 1967; Prodanović et al., 2004; } \\
\text { Prodanović, } 2007\end{array}$ & hairy melic, silky spike melic \\
\hline Melica nutans L. & \begin{tabular}{|l|} 
Prodanović, 2007 \\
\end{tabular} & mountain melick \\
\hline Melica uniflora Retz. & Prodanović, 2007 & wood melick \\
\hline Milium effusum $\mathrm{L}$. & Prodanović, 2007 & $\begin{array}{c}\text { american milletgrass, wood } \\
\text { millet }\end{array}$ \\
\hline Phalaris arundinacea $\mathrm{L}$. & Prodanović, 2007 & reed canary grass \\
\hline Phleum montanum K.Koch & Prodanović, 2007 & Timothy grass \\
\hline Phleum phleoides (L.) H. Karst. & Prodanović, 2007 & $\begin{array}{c}\text { boehmer's cat's-tail, purple-stem } \\
\text { cat's-tail }\end{array}$ \\
\hline Phleum pratense L. & Prodanović, 2007 & Timothy grass, meadow cat's-tail \\
\hline $\begin{array}{l}\text { Phragmites australis (Cav.) Trin. ex } \\
\text { Steud. }\end{array}$ & Prodanović, 2007 & common reed \\
\hline Poa anпua L. & Prodanović, 2007 & annual meadow grass \\
\hline Poa bulbosa L. & Prodanović, 2007 & bulbous bluegrass \\
\hline Poa compressa $\mathrm{L}$. & Prodanović, 2007 & canada bluegrass \\
\hline Poa nemoralis L. & Prodanović, 2007 & wood bluegrass \\
\hline Poa pratensis $\mathrm{L}$. & Prodanović, 2007; Krivošej et al., 2013 & kentucky bluegrass \\
\hline Poa perconcinna J. R. Edm. & Prodanović, 2007 & \\
\hline Poa trivialis $\mathrm{L}$. & Prodanović, 2007 & rough bluegrass \\
\hline Sclerochloa dura (L.) P. Beauv. & Prodanović, 2007 & common hardgrass \\
\hline Sesleria rigida Heuff. ex Rchb. & Prodanović, 2007 & \\
\hline Stipa capillata $\mathrm{L}$. & Prodanović, 2007 & needle grass \\
\hline Stipa joannis Celak & Prodanović, 2007 & feather grass \\
\hline Taeniatherum caput-medusae (L.) Nevski & Prodanović, 2007 & medusahead wildrye \\
\hline Tragus racemosus (L.) All. & $\begin{array}{c}\text { Krivošej et al. 1995-1998; Prodanović, } \\
2007\end{array}$ & stalked bur grass \\
\hline Vulpia myuros (L.) C.C.Gmel. & Prodanović, 2007 & annual fescue \\
\hline \multicolumn{3}{|l|}{ POLYGALACEAE } \\
\hline Polygala major Jacq. & Prodanović, 2007 & milkworts, snakeroots \\
\hline Polygala supina Schreb. & Pavlović, 1967; Prodanović, 2007 & milkwort \\
\hline
\end{tabular}




$$
-7311 \text { - }
$$

\begin{tabular}{|c|c|c|}
\hline CLASS/ FAMILY/Species & Source/Literature & Common English names \\
\hline Fallopia convolvulus (L.) Á. Löve & Prodanović, 2007 & black-bindweed, wild buckwheat \\
\hline Fallopia dumetorum (L.) Holub & Prodanović, 2007 & european climbing buckwheat \\
\hline Persicaria lapathifolia (L.) Delarbre & Prodanović, 2007 & pale persicaria \\
\hline Polygonum aviculare $\mathrm{L}$. & Prodanović, 2007 & common knotgrass \\
\hline Rumex acetosa $\mathrm{L}$. & Prodanović, 2007; Krivošej et al., 2013 & sorrel \\
\hline Rumex acetosella $\mathrm{L}$. & Prodanović, 2007 & red sorrel, sheep's sorrel \\
\hline Rumex conglomeratus Murray & Prodanović, 2007 & clustered dock, sharp dock \\
\hline Rumex crispus L. & Prodanović, 2007 & curled dock, yellow dock \\
\hline Rumex obtusifolius L. & Prodanović, 2007 & bitter dock \\
\hline Rumex pulcher L. & Prodanović, 2007 & fiddle dock \\
\hline \multicolumn{3}{|l|}{ PRIMULACEAE } \\
\hline Anagallis arvensis $\mathrm{L}$. & Prodanović, 2007 & scarlet pimpernel \\
\hline Anagallis foemina Mill. & Prodanović, 2007 & blue pimpernel \\
\hline Androsace elongata $\mathrm{L}$. & Prodanović et al.,2012 & rock jasmine, elongate ilisha \\
\hline Lysimachia nummularia L. & Prodanović, 2007; Krivošej et al., 2013 & moneywort, creeping jenny \\
\hline Primula veris Huds. & Prodanović, 2007 & ommon cowslip \\
\hline Primula acaulis (L.) L. & Krivošej et al., 2013 & common primrose \\
\hline \multicolumn{3}{|l|}{ RANUNCULACEAE } \\
\hline Anemone apennina $\mathrm{L}$. & Prodanović, 2007 & blue anemone \\
\hline Anemone nemorosa L. & Prodanović, 2007 & wood anemone \\
\hline Clematis recta $\mathrm{L}$. & Prodanović, 2007 & $\begin{array}{c}\text { erect clematis, ground } \\
\text { virginsbower }\end{array}$ \\
\hline Consolida regalis Gray & Prodanović, 2007 & forking larkspur, rocket-larkspur \\
\hline Ficaria verna Huds. & $\begin{array}{c}\text { Prodanović (unpublished, field } \\
\text { observation) }\end{array}$ & fig buttercup \\
\hline $\begin{array}{l}\text { Helleborus odorus Waldst. \& Kit. ex } \\
\text { Willd. }\end{array}$ & $\begin{array}{l}\text { Prodanović et al., 2004; Prodanović, } \\
2007\end{array}$ & fragrant hellebore \\
\hline $\begin{array}{c}\text { Helleborus multifidus } \\
\text { subsp. serbicus (Adamovic) Merxm.\& } \\
\text { Podlech } \\
\end{array}$ & Prodanović, 2007 & hellebore \\
\hline Hepatica nobilis Mill. & Prodanović, 2007 & common hepatica, liverwort \\
\hline Nigella arvensis L. & Prodanović, 2007 & love-in-a-mist, black bread-weed \\
\hline $\begin{array}{c}\text { Pulsatilla vulgaris } \\
\text { subsp. grandis (Wender) Zämelis }\end{array}$ & Prodanović, 2007 & pasqueflower \\
\hline Ranunculus arvensis L. & Prodanović, 2007 & corn buttercup \\
\hline Ranunculus bulbosus L. & Prodanović, 2007 & bulbous buttercup \\
\hline Ranunculus millefoliatus Vahl & Prodanović, 2007; Krivošej et al., 2013 & erusalem butercup \\
\hline Ranunculus polyanthemos $\mathrm{L}$. & Prodanović, 2007 & multiflowered buttercup \\
\hline Ranunculus psilostachys Griseb. & Prodanović, 2007 & buttercup \\
\hline Ranunculus repens $\mathrm{L}$. & Prodanović, 2007 & the creeping buttercup \\
\hline Ranunculus sceleratus L. & Prodanović, 2007 & celery-leaved buttercup \\
\hline Ranunculus serbicus Vis. & Prodanović, 2007 & \\
\hline Thalictrum flavum $\mathrm{L}$. & Prodanović, 2007 & common meadow-rue \\
\hline Thalictrum lucidum $\mathrm{L}$. & Prodanović, 2007 & shaning meadow rue \\
\hline Thalictrum minus L. & Prodanović, 2007 & lesser meadow-rue \\
\hline \multicolumn{3}{|l|}{ RESEDACEAE } \\
\hline Reseda lutea $\mathrm{L}$. & Prodanović, 2007 & wild mignonette \\
\hline Reseda luteola $\mathrm{L}$. & Prodanović, 2007 & $\begin{array}{c}\text { dyer's weed, weld, woold, yellow } \\
\text { weed }\end{array}$ \\
\hline Reseda phyteuma L. & Prodanović, 2007 & rampion mignonette \\
\hline \multicolumn{3}{|l|}{ RHAMNACEAE } \\
\hline Frangula alnus Mill. & Prodanović, 2007 & alder buckthorn \\
\hline Paliurus spina-christi Mill. & Prodanović, 2007 & Jerusalem thorn \\
\hline $\begin{array}{c}\text { Rhamnus saxatilis } \\
\text { subsp. tinctoria } \text { Nyman }\end{array}$ & Prodanović, 2007 & rock buckthorn \\
\hline \multicolumn{3}{|l|}{ ROSACEAE } \\
\hline Agrimonia eupatoria L. & Prodanović, 2007 & agrimony, cocklebur \\
\hline
\end{tabular}




$$
-7312 \text { - }
$$

\begin{tabular}{|c|c|c|}
\hline CLASS/ FAMILY/Species & Source/Literature & Common English names \\
\hline Amelanchier ovalis Medik. & Prodanović, 2007 & snowy mespilus \\
\hline Aremonia agrimonioides (L.) DC. & Krivošej et al., 2013 & bastard-agrimony \\
\hline Cotoneaster integerrimus Medik. & Prodanović, 2007 & cotoneaster \\
\hline Crataegus monogyna Jacq. & $\begin{array}{c}\text { Prodanović et al., 2004; Prodanović, } \\
2007\end{array}$ & ommon hawthorn \\
\hline Filipendula vulgaris Moench. & Prodanović, 2007; Krivošej et al., 2013 & dropwort,fern-leaf dropwort \\
\hline Fragaria vesca $\mathrm{L}$ & $\begin{array}{c}\text { Prodanović et al., 2004; Prodanović, } \\
\text { 2007; Krivošej et al., } 2013\end{array}$ & wild strawberry \\
\hline Geum urbanum L. & Prodanović, 2007; Krivošej et al., 2013 & wood avens \\
\hline $\begin{array}{l}\text { Malus florentina (Zuccagni) C.K. } \\
\text { Schneid. }\end{array}$ & Prodanović et al., 2013 & florentine crabapple \\
\hline Malus sylvestris (L.) Mill. & Prodanović, 2007 & european crab apple \\
\hline Potentilla argentea $\mathrm{L}$. & $\begin{array}{l}\text { Prodanović et al., 2004; Prodanović, } \\
2007\end{array}$ & $\begin{array}{c}\text { hoary cinquefoil, } \\
\text { silver cinquefoil }\end{array}$ \\
\hline $\begin{array}{c}\text { Potentilla heptaphylla } \\
\text { subsp. australis (Nyman) Gams }\end{array}$ & $\begin{array}{l}\text { Prodanović et al., 2004; Prodanović, } \\
2007 \\
\end{array}$ & \\
\hline $\begin{array}{c}\text { Potentilla hirta L. } \\
\text { var. zlatiborensis Novak }\end{array}$ & Rexhepi, 1979; Prodanović, 2007 & hairy cinquefoil \\
\hline Potentilla micrantha Ramond ex DC & Prodanović, 2007 & pink barren strawberry \\
\hline Potentilla neglecta Baumg. & Prodanović, 2007 & \\
\hline Potentilla recta $\mathrm{L}$. & Prodanović, 2007 & sulfur cinquefoil \\
\hline Potentilla tommassiniana F. W. Schultz. & Pavlović, 1967 & \\
\hline Potentilla visianii Pančić & $\begin{array}{l}\text { Rexhepi, 1979; Prodanović, 2007; } \\
\text { Prodanović et al., } 2008\end{array}$ & \\
\hline Prunus avium (L.) L. & $\begin{array}{l}\text { Prodanović et al., 2004; Prodanović, } \\
2007 \\
\end{array}$ & wild cherry \\
\hline Prunus mahaleb L. & Prodanović, 2007 & mahaleb cherry \\
\hline $\begin{array}{c}\text { Prunus spinosa } \\
\text { subsp. dasyphylla (Schur) Domin }\end{array}$ & Prodanović, 2007 & blackthorn, sloe \\
\hline $\begin{array}{c}\text { Prunus spinosa L. } \\
\text { subsp. spinosa }\end{array}$ & $\begin{array}{c}\text { Prodanović et al., 2004; Prodanović, } \\
2007 \\
\end{array}$ & blackthorn, sloe \\
\hline Pyrus spinosa Forssk. & Prodanović, 2007 & almond-leaved pear \\
\hline Rosa arvensis Huds. & Prodanović, 2007 & field rose \\
\hline Rosa canina $\mathrm{L}$. & Prodanović, 2007 & dog rose \\
\hline Rosa corymbifera Borkh. & Prodanović, 2007 & rose \\
\hline Rosa $x$ dumetorum Thuill & Prodanović, 2007 & corymb rose \\
\hline Rosa gallica $\mathrm{L}$. & Prodanović, 2007; Krivošej et al., 2013 & french rose \\
\hline Rosa micrantha Borrer ex Sm. & Prodanović, 2007 & rugosa rose \\
\hline Rosa rubiginosa $\mathrm{L}$. & Prodanović, 2007 & sweetbriar rose \\
\hline Rosa spinosissima $\mathrm{L}$. & Prodanović, 2007 & scotch rose \\
\hline Rubus caesius L. & Prodanović, 2007 & european dewberry \\
\hline Rubus ulmifolius Schott & Prodanović, 2007 & elm-leaf blackberry \\
\hline Sanguisorba minor Scop & Prodanović, 2007; Krivošej et al., 2013 & salad burnet \\
\hline Sanguisorba officinalis L. & Prodanović, 2007 & great burnet \\
\hline Spiraea media Schmidt & Prodanović, 2007 & spirea \\
\hline Sorbus aucuparia L. & Prodanović, 2007 & mountain ash \\
\hline Sorbus torminalis (L.) Crantz & Prodanović, 2004; Prodanović, 2007 & wild service tree, chequers \\
\hline Waldsteinia geoides Willd. & Prodanović et al.,2010 & barren strawberries \\
\hline \multicolumn{3}{|l|}{ RUBIACEAE } \\
\hline Asperula cynanchica $\mathrm{L}$. & Rexhepi, 1979; Prodanović, 2007 & squinancywort, squincywort \\
\hline Asperula purpurea (L.) Ehrend. & Prodanović, 2007 & purple squinancywort \\
\hline Cruciata glabra (L.) Opiz. & Prodanović, 2007 & \\
\hline Cruciata laevipes Opiz. & Prodanović, 2007; Krivošej et al., 2013 & crosswort smooth bedstraw \\
\hline $\begin{array}{l}\text { Cruciata pedemontana (Bellardi) Ehrend., } \\
\text { All. }\end{array}$ & Prodanović, 2007 & Piedmont bedstraw \\
\hline Galium aparine L. & Prodanović, 2007 & catchweed bedstraw \\
\hline Galium mollugo L. & Prodanović, 2007 & hedge bedstraw \\
\hline
\end{tabular}




$$
-7313-
$$

\begin{tabular}{|c|c|c|}
\hline CLASS/ FAMILY/Species & Source/Literature & Common English names \\
\hline Galium pseudoaristatum Schur & Prodanović, 2007 & bedstraw \\
\hline Galium verum L. & Prodanović, 2007 & yellow bedstraw \\
\hline Sherardia arvensis L. & Prodanović, 2007 & blue fieldmadder \\
\hline \multicolumn{3}{|l|}{ RUTACEAE } \\
\hline Dictamnus albus L. & Prodanović, 2007 & burning bush, dittany \\
\hline $\begin{array}{c}\text { Haplophyllum boissierianum Vis. et } \\
\text { Pančić }\end{array}$ & $\begin{array}{c}\text { Pavlović, 1967; Ranđelović et al., 1982; } \\
\text { Prodanović, 2007; Prodanović et al., } \\
2008\end{array}$ & \\
\hline \multicolumn{3}{|l|}{ SALICACEAE } \\
\hline Populus alba L. & Prodanović, 2007 & silver poplar, silverleaf poplar \\
\hline Populus nigra L. & Prodanović, 2007 & black poplar \\
\hline Populus tremula $\mathrm{L}$. & Prodanović, 2007; Krivošej et al., 2013 & aspen, common aspen \\
\hline Salix alba $\mathrm{L}$ & Prodanović, 2007 & golden willow, white willow \\
\hline Salix caprea $\mathrm{L}$. & Prodanović, 2007 & goat willow, pussy willow \\
\hline Salix eleagnos Scop. & Prodanović, 2007 & bitter willow, olive willow \\
\hline Salix $x$ fragilis L. & Prodanović, 2007 & crack willow \\
\hline Salix purpurea L. & Prodanović, 2007 & purple willow \\
\hline \multicolumn{3}{|l|}{ SANTALACEAE } \\
\hline Arceuthobium oxycedri (DC.) M. Bieb. & Ranđelović et al., 1982; Prodanović 2007 & juniper dwarf mistletoe \\
\hline $\begin{array}{c}\text { Comandra umbellata } \\
\text { subsp. elegans (Rochel ex Rchb.) Piehl }\end{array}$ & Prodanović, 2007 & bastard toadflax \\
\hline Thesium arvense Horv. & Rexhepi, 1979; Prodanović, 2007 & \\
\hline \multicolumn{3}{|l|}{ SAPINDACEA } \\
\hline Acer campestre L. & $\begin{array}{l}\text { Prodanović et al., 2004; Prodanović, } \\
2007 \\
\end{array}$ & field maple \\
\hline $\begin{array}{c}\text { Acer campestre } \\
\text { subsp. marsicum (Guss.) Hayek }\end{array}$ & Prodanović, 2007 & field maple \\
\hline Acer platanoides L. & Prodanović, 2007 & $\begin{array}{c}\text { emerald queen maple, norway } \\
\text { maple }\end{array}$ \\
\hline Acer pseudoplatanus L. & Prodanović, 2007 & sycamore \\
\hline Acer tataricum $\mathrm{L}$. & Prodanović, 2007 & tatarian maple \\
\hline \multicolumn{3}{|l|}{ SAXIFRAGACEAE } \\
\hline Saxifraga paniculata Mill. & Rexhepi, 1979; Prodanović, 2007 & alpine saxifrage \\
\hline Saxifraga bulbifera $\mathrm{L}$. & Prodanović, 2007 & bulbous saxifrage \\
\hline Saxifraga rotundifolia $\mathrm{L}$. & Prodanović, 2007 & round-leaved saxifrage \\
\hline Saxifraga tridactylites $\mathrm{L}$. & Prodanović, 2007 & rue-leaved saxifrage \\
\hline \multicolumn{3}{|l|}{ SCROPHULARIACEAE } \\
\hline $\begin{array}{c}\text { Scrophularia canina } \\
\text { subsp. bicolor }(\mathrm{Sm} .) \text { Greuter }\end{array}$ & Prodanović, 2007 & dog figwort \\
\hline Scrophularia canina L. & $\begin{array}{l}\text { Ranđelović et al., 1982; Prodanović, } \\
2007\end{array}$ & dog figwort \\
\hline $\begin{array}{c}\text { Scrophularia canina } \mathrm{L} . \\
\text { subsp. tristis (K. Malý) V. Nikolic }\end{array}$ & Pavlović, 1967; Prodanović, 2007 & dog figwort \\
\hline Scrophularia nodosa L. & Prodanović, 2007 & figwort, woodland figwort \\
\hline Scrophularia umbrosa Dumort. & Prodanović, 2007 & green figwort \\
\hline Verbascum banaticum Schrad. & Prodanović, 2007 & mullein \\
\hline Verbascum chaixii Vill. & Prodanović, 2007 & narrow-leaved mullein \\
\hline Verbascum nigrum L. & Prodanović, 2007 & black mullein, dark mullein \\
\hline Verbascum phlomoides L. & Prodanović, 2007 & orange mullein \\
\hline Verbascum phoeniceum L. & Prodanović, 2007 & purple mullein \\
\hline \multicolumn{3}{|l|}{ SOLANACEAE } \\
\hline Datura stramonium L. & Prodanović, 2007 & thorn apple, jimsonweed \\
\hline Hyoscyamus niger L. & Prodanović, 2007 & henbane, black henbane \\
\hline Lycium barbarum $\mathrm{L}$. & Prodanović, 2007 & $\begin{array}{c}\text { chinese boxthorn, Himalayan } \\
\text { goji }\end{array}$ \\
\hline Solanum dulcamara L. & Prodanović, 2007 & $\begin{array}{l}\text { bittersweet, bittersweet } \\
\text { nightshade }\end{array}$ \\
\hline
\end{tabular}




\begin{tabular}{|c|c|c|}
\hline CLASS/ FAMILY/Species & Source/Literature & Common English names \\
\hline \multicolumn{3}{|l|}{ THYMELAEACEAE } \\
\hline Thymelaea passerina (L.) Coss. \& Germ. & Prodanović et al.,2010 & sparrow-wort \\
\hline \multicolumn{3}{|l|}{ ULMACEAE } \\
\hline Ulmus carpinifolia Gled. & Prodanović, 2007 & elm \\
\hline Ulmus laevis Pall. & Prodanović, 2007 & $\begin{array}{l}\text { european white elm, } \\
\text { fluttering elm }\end{array}$ \\
\hline Ulmus minor Mill. & $\begin{array}{c}\text { Prodanović et al., 2004; Prodanović, } \\
2007\end{array}$ & elm \\
\hline $\begin{array}{c}\text { Ulmus minor Mill } \\
\text { var. tortuosa (Host) Hayek }\end{array}$ & Prodanović, 2007 & elm \\
\hline \multicolumn{3}{|l|}{ URTICACEAE } \\
\hline Parietaria officinalis L. & Prodanović, 2007 & eastern pellitory-of-the-wall \\
\hline Urtica dioica $\mathrm{L}$. & Prodanović, 2007 & common nettle, stinging nettle \\
\hline \multicolumn{3}{|l|}{ VERBENACEAE } \\
\hline Verbena officinalis L. & Prodanović, 2007 & common vervain \\
\hline \multicolumn{3}{|l|}{ VIBURNACEAE } \\
\hline Sambucus ebulus L. & Prodanović, 2007 & danewort, dane weed \\
\hline Sambucus nigra L. & Prodanović, 2007 & elderberry, black elder \\
\hline \multicolumn{3}{|l|}{ VIOLACEAE } \\
\hline Viola arvensis Murray & $\begin{array}{l}\text { Ranđelović et al., 1982; Prodanović, } \\
2007\end{array}$ & field pansy \\
\hline Viola hirta L. & Prodanović, 2007 & sweet violet, english violet \\
\hline Viola kitaibeliana Schult. & Prodanović, 2007 & Kitaibel's violet \\
\hline Viola reichenbachiana Boreau & Prodanović, 2007 & $\begin{array}{c}\text { early dog-violet, pale wood } \\
\text { violet }\end{array}$ \\
\hline
\end{tabular}

A vast majority of genera belong to the familiy Compositae (51), followed by Poaceae (38), Apiaceae (26), Brassicaceae (24), Leguminosae (24), Lamiaceae (22). Among the genera the most species rich are Trifolium (17) followed by Carex (16), Euphorbia (14), Veronica (14), Vicia (13), Lathyrus (12), Hieracium (11), Centaurea (8), Medicago (8), Rosa (7) etc. The dominance of the genera Trifolium, Vicia and Lathyrus (Leguminosae family) is most likely conditioned by the higher presence of areas under xerothermic meadows and pastures as well as thermophilic rocks in the study area. Genus Carex is on second position. This is explained by the fact that species within this genus require moist habitats, which were abundant in the investigated terrains, on the banks of river Ibar, as well as streams flowing into the river. Genera Trifolium and Carex are also dominant in serpentine complexes in Eastern Rhodopes (Bulgaria) (Pavlova et al., 2004).

31 taxa in total have been recorded in this area for the first time and they present a novelty for the flora of Kosovo and Metohija and entire Serbia. The records for most of these were first published in Prodanović et al. (2004, 2008, 2010, 2012, 2013, 2018).

\section{Life-form spectrum and phytogeographical analysis}

The analysis of life forms indicates domination of hemicryptophytes (48.29\%) (Fig. 2). The significant representation of therophytes (21.99\%), is typical for the serpentine floras, where the plants are adapted to ensure reproduction within a short period of time under stress conditions (Brooks, 1987). Other herbal life forms are present in smaller numbers, geophytes $(10.54 \%)$ phanerophytes $(8.27 \%)$, chamaephytes $(6.91 \%)$, scandentophytes $(1.81 \%)$ etc. Such biological spectrum is characteristic for the Balkan Peninsula, as well as for the territory of Serbia (Diklić, 1984). 


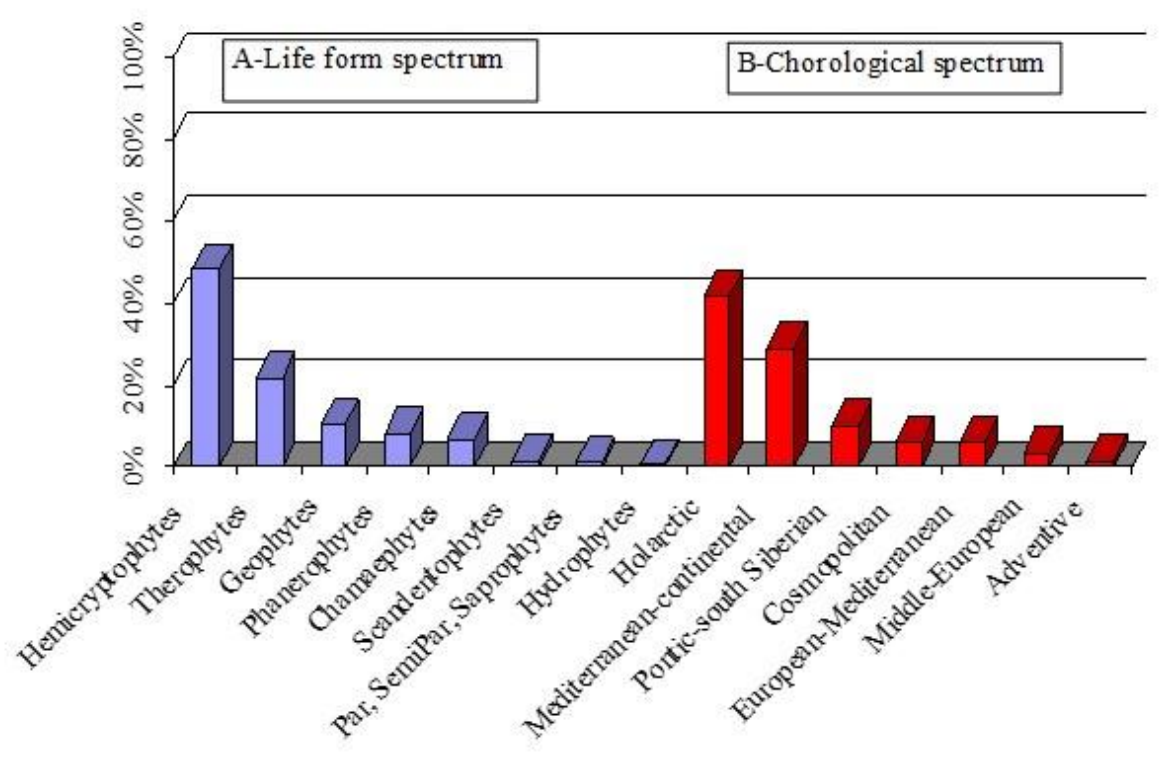

Figure 2. Life form spectrum (A) and chorological spectrum (B), by basic area types, of the serpentinite flora in the middle course of the Ibar river valley

The chorological floristic analysis indicates that this is an area with interim holarcticMediterranean character (Fig. 2). Holarctic areal type with 369 taxa (41.83\%) is the most presented on the terrain, followed by Mediterranean - continental areal type $(29.18 \%)$ and pontic - south Siberian areal type (10.31\%), cosmopolitan areal type $(6.45 \%)$ as well as central European -Mediterranean (6.23\%). The least reported species in the serpentine terrains of the Ibar river valley are Middle European dispersion areal type and Adventive areal type. The presence of plants belonging to Adventive areal type indicates anthropogenic influence, which is present in settlements around river banks, as well as around main roads and local rural roads. Xeromorphic species are predominant due to conditions for their growth on serpentine soil.

\section{Endemism and internationally significant vascular plants}

Serpentine habitats are the most important endemic regions in the world and also called "geological islands" (Kurt et al., 2013). Despite the shallow active layer of serpentine soil, biodiversity is high with a great number of interesting local and regional endemics (Shuka and Hallaçi, 2010). It is estimated that the total number of endemic plants in the Balkans is bigger than 2200 taxa. 300-350 (15-16\%) taxa of this number have been identified on the serpentine background; 123 of them are obligate serpentine endemics (Stevanović et al., 2003).

Due to diverse physical and geographical characteristics, the Republic of Serbia has a considerable number of endemic plant taxa. Centres of endemism are located in the southern and eastern regions of Serbia and on the territory of Kosovo and Metohija (Gavrilović et al., 2017). The serpentine mountains of Central Serbia along the river Ibar represent a relatively large core of ophiolithic flora in the Central Balkans. It is the centre of distribution of old trans-regional endemics such as: Halacsya sendtneri, Haplophyllum boisserianum, Potentilla visianii and Eryngium serbicum. Local endemics include Tulipa serbica (Stevanović et al., 2003). 
Out of the total taxon number in the investigated area, $73(8.27 \%)$ belong to group of endemic, sub-endemic, relict and endomorelic taxa. There are 40 of endemic and subendemic species (Eryngium serbicum, Helleborus multifidus subsp. serbicus, Tulipa serbica, Odontarrhena bertolonii subsp. scutarina (syn. Alyssum janchenii), Alyssum markgrafii, Galatella albanica, Dianthus pinifolius, Euphorbia glabriflora, Fumana bonapartei, Halacsya sendtneri, Haplophyllum boissierianum, Hieracium bauhini subsp. pseudosparsum, Hypericum rumeliacum, Lamium bifidum subsp. balcanicum, Potentilla visianii, Ranunculus psilostachys, Scabiosa fumaroides, Scrophularia canina subsp. tristis, Sedum album (syn. S. serpentini), Stachys scardica, Linum flavum, Trifolium trichopterum, Rorippa lippizensis, Salvia amplexicaulis, Digitalis laevigata, Melampyrum heracleoticum, Eryngium palmatum, Achillea coarctata, Alyssum montanum subsp. serbicum, Campanula lingulata, Cytisus austriacus subsp. heuffelii, Galium pseudoaristatum, Iris reichenbachii, Lamium garganicum L. subsp. garganicum, Lamium garganicum L. subsp. glabratum, Lathyrus hallersteinii, Petrorhagia illyrica subsp. haynaldiana, Poa perconcinna, Ranunculus serbicus, Rhinanthus rumelicus, Trifolium pignantii).

Based on the distributional range of obligate serpentine endemics, Stevanović et al. (2003) presented the following general classification: (1) trans-Balkan or trans-regional Balkan endemics (taxa distributed in the greater part of serpentine areas in the Balkans); (2) regional endemics (taxa restricted to a single floristic subregion or province); and (3) local or steno-endemics (taxa distributed in a single floristic district or narrow geographical area such as a single mountain or island).

On the investigated terrains in the middle course of the Ibar river valley, 11 taxa from obligate serpentine endemics are in the trans-regional endemic (TRE) category : Alyssum markgrafii, Sedum album (syn. S. serpentini), Potentilla visianii, Potentilla heptaphylla subsp. australis, Euphorbia glabriflora, Haplophyllum boissierianum, Fumana bonapartei, Eryngium serbicum, Halacsya sendtneri, Stachys recta subsp. baldacii and Scrophularia canina subsp. tristis.

4 taxa are obligate serpentine endemics from the regional endemics (RE) category: Helleborus multifidus subsp. serbicus, Alyssum montanum subsp. serbicum, Galatella albanica and Tulipa serbica (stenoendemic according to Millaku et al., 2018). It was right here, on the Ibar Valley serpentines, Beli Laz locality (locus classicus), that this endemic Tulip was identified (Tatić and Krivošej, 1997). Unfortunately, in the last three years, due to the regional dumping site development, the primary habitat has been devastated, without any possibility to be protected.

Out of the total taxon number, 31 taxa belong to a group of internationally significant vascular plants (IUCN, Stojanović et al., 2015). Out of that, 14 taxa have been protected by CITES Convention. These are the followings: Anacamptis pyramidalis, Cephalanthera rubra, Epipactis helleborine, Epipactis microphylla, Galanthus nivalis, Limodorum abortivum, Neottia nidus-avis, Orchis mascula, Orchis morio, Orchis papilionaceae, Orchis purpurea, Orchis tridentata, Ophrys apifera and Platanthera bifolia.

\section{Comparative floristic analysis}

In order to gain a better insight into the flora diversity in these habitats, as well as the level of floristic closeness, a comparative analysis of the flora from Studena mountain, near the city of Kraljevo, has been done by Tatić (1969) in his multi-year research as well as with flora of Goleš mountain, researched by Krasniqi et al. (2019). 
The Ibar serpentine massive with the valley in the lower course of the Ibar river traverses though central Serbia and extend west from the mountain Studena (Lukić et al., 2015) where it covers the largest space on Studena mountain; the river itself flows $17.1 \mathrm{~km}$ along the mountain. In his studies Tatić reported 390 taxa, the largest number of which belonged to Compositae, Poaceae, Leguminosae, Caryophyllaceae, Rosaceae and Lamiaceae families, which is similar to the spectrum of the most numerous families of flora in the Kosovo part of the Ibar serpentine range. The number of two mutual taxa in two compared areas is 196, so the index of similarity by Sørensen is $30.66 \%$, which is unexpectedly low since Studena mountain represents the continuation of the Ibar serpentine massive that starts in the middle course of the river in Kosovo.

An assumption of floristic dissimilarities in the Kosovo part of the Ibar valley and Studena mountain, has been based on the differences between the altitudes of these two areas (the Ibar gorge 500-900 ma, Studena mountain above 1000 ma, with the highest peak of Kavgalija 1356). Furthermore, the composition of the geological background has probably influenced floral development. The geological background of Studena mountain exclusively consists of serpentinite; in the Kosovo part of the Ibar valley apart from serpentinite, there are also peridotite and unmodified ultramaphyte rocks. While Tatić emphasized phytocenologic research studies on Studena mountain, our attention was turned to purely floristic research projects in the Kosovo's part of the Ibar valley (which may be corroborated by a total number of 882 identified taxa), taking also into account the size of the investigated area. Since the serpentine is considered as a vulnerable habitat, it is important to emphasize that no Studena mountain species recorded by Tatic has been classified as extinct in Serbian flora. Our assumption is that the described species can be found there nowadays, but we also recommend future field research to prove this assumption.

Mountain Goleš, in the central part of Kosovo and Metohija, represents the beginning of the serpentine-peridotite massif, which covers the left bank of the Sitnica river, goes towards the Čičavica Mountain, enters the valley of the upper stream of the Ibar river in Ibarski Kolašin, and continues along the middle Ibar river course. Krasniqi et al. (2019) recorded 295 taxa on this mountain (with a surface area of $22.2 \mathrm{~km}^{2}$ ) over a three-year study (2015-2019). Considering the size of the mountain range covered by the conducted survey, a larger number of taxa would be expected. However, such floristic "poverty" confirmed the fact that serpentinite habitats are floristically poorer than habitats with other types of geological substrates. The number of two mutual taxa in the two compared areas is 218 , so the index of similarity by Sørensen is $37.04 \%$. It should be noted that this value is not large, although the number of common species is as high as 2/3 of all taxa found on Goleš Mountain. The reason could certainly be a disproportion to the number of identified species and the size of territories explored.

Families with the highest number of taxa on Goleš mountain were Compositae (34), Leguminosae (25), Rosaceae (25), Poaceae (18), and Caryophyllaceae (18). In taxonomic spectrum in the Ibar river valley, Compositae (105) and Leguminosae (86) are also dominant, followed with Poaceae (66), Lamiaceae (51), Brassicaceae (50), Caryophyllaceae (49). Rosaceae family in taxonomic spectrum of most dominant plant families in the Ibar river valley occupies eight position. Significant involment of the Lamiaceae and Caryophyllaceae families indicate the expression of stronger Mediterranean floristic influences, as well as a greater presence of bare rocky habitats.

Krasniqi et al. (2019) on Goleš Mountain state that out of the total number of identified taxa, 8 of them are endemic, of which 5 endemic species occur on the Ibar 
valley serpentinite (Galatella albanica, Halacsya sendtneri, Linum flavum, Potentilla visianii and Haplophyllum boissierianum). Various floristic elements meet and overlap in mountain ranges in the central part of Kosovo, dominated by European floral element, followed by Euro-Asiatic and Sub-Mediterranean, Balcan, Mediterranean and Pontic elements similar to the interim holarctic- Mediterranean character of flora in the Ibar river valley.

Due to the background characteristics, the serpentine areas of the Ibar valley and their flora have not been exposed to negative anthropogenic influences so far. Yet, in the previous 3-4 years, the negative human influences have been detected in the areas along the highway. We consider that establishing a better connection between scientific and professional public and the people who are in charge of planning and infrastructural construction along the Ibar valley could contribute to the preservation of the habitats of some significant and endangered species, which unfortunately was not the case with locus classicus of the Tulipa serbica species.

\section{Conclusion}

The presence of 882 taxa grouped into 83 families and 386 genera has been identified in the course of the research of serpentinite flora performed in the middle stream Ibar river valley, for the period of 16 years. Taxonomic spectrum of families is dominated by Compositae (105), followed by Leguminosae (86), Poaceae (66), Lamiaceae (51), Brassicaceae (50), Caryophyllaceae (49), Apiaceae (43) etc. The greatest number of genera belong to families Compositae (51), followed by Poaceae (38), Apiaceae (26), Brassicaceae (24), Leguminosae (24), Lamiaceae (22). Among the genera the most species rich are Trifolium (17) followed by Carex (16), Euphorbia (14), Veronica (14), Vicia (13), Lathyrus (12), Hieracium (11), Centaurea (8), Medicago (8), Rosa (7) etc. An analysis of life forms showed that the investigated area has hemicryptophyte characters, with significant participation of therophytes $(21.99 \%)$. The chorological spectrum is dominated by Holarctic species $(41.83 \%)$. The presence of 73 taxa from a group of endemic, sub-endemic, relict and endomorelic taxa was determined. Out of the total taxon number, 31 taxa belong to a group of internationally significant vascular plants. Out of that, 14 taxa have been protected by CITES Convention. A comparative analysis of the serpentinite flora in the Ibar river valley and the Studena mountain as well as in the Goleš mountain showed a relatively small floristic similarity, which was unexpected, since these two mountains represent the beginning and continuation of the Ibar serpentine massive that starts in the middle course of the river in Kosovo. Only a good knowledge of floristic diversity may initiate the procedures that may lead to preserving and protecting rare and endangered and internationally important species in these areas. Since serpentinite habitat is considered as vulnerable we are proposing continual habitat monitoring as well as monitoring of all endangered and rare species in the Ibar river valley in future research projects. For some species seed collecting for a seed bank could be one of the conservation measures.

Acknowledgements. The authors are grateful to dr Marjan Niketić, from the Natural History Museum in Belgrade, for his help in the determination of genus Hieracium and Cerastium. 


\section{REFERENCES}

[1] Anacker, B. L. (2014): The nature of serpentine endemism. - American Journal of Botany 101(2): 219-224.

[2] Bani, A., Imeri, A., Echevarria, G., Pavlova, D., Reeves, R., Morel, J. L., Sulçe, S. (2013): Nickel hyperaccumulation in the serpentine flora of Albania. - Fresenius Environmental Bulletin 22(6): 1792-1801.

[3] Brady, K., Kruckeberg, A., Bradshaw Jr., H. D. (2005): Evolutionary Ecology of Plant Adaptation to serpentine Soils. - Annu. Rev. Ecol. Evol. Syst. 36: 243-266.

[4] Branković, S., Cupara, S., Glišić, R., Djelić, G., Grbović, F., Kojičić, K., Milovanović, O. (2017): Phytoaccumulation in plants of Mountain Goc in Serbia. - Studia Universitatis "Vasile Goldiş", Seria Ştiinţele Vieţii 27(3): 196-201.

[5] Brooks, R. R. (1987): Serpentine and its Vegetation: A Multidisciplinary Approach. Dioscorides Press, Portland, Oregon.

[6] CITES Secretariat: Convention on International Trade in Endangered Species of Wild Fauna and Flora. - http://www.checklist.cites.org.

[7] Diklić, N. (1984): Life forms and biology spectrum of flora of SR Serbia. - In: Sarić, M. (ed.) Vegetation of Serbia 1. SANU, Belgrade. (Životne forme biljnih vrsta i biološki spektar flore SR Srbije. - In: Vegetacija Srbije 1, Opšti deo, Sarić M. (ed.), SANU, Beograd) (in Serbian).

[8] Dudić, B., Rakić, T., Šinžar-Sekulić, J., Atanacković, V., Stevanović, B. (2007): Differences of metal concentrations and morpho-anatomical adaptations between obligate and facultative serpentinophytes from Western Serbia. - Arch. Biol. Sci. 59(4): 341-349.

[9] Gavrilović, B., Tomović, G., Niketić, M., Milenković, M., Simić, S., Radovanović, M. (2017): Geoecological characteristics of plant endemism in the Balkan part of Serbia. Botanica Serbica 41(2): 177-197.

[10] Guillot, S., Hattori, K. (2013): Serpentinites: essential roles in geodynamics, arc volcanism, sustainable development, and the origin of life. - Elements 9: 95-98.

[11] Harrison, S., Rajakaruna, N. (2011): Serpentine: The evolution and Ecology of a Model System. - University of California Press, Berkley and Los Angeles.

[12] International Union for Conservation of Nature. http://www.iucn.org/about/union/secretariat/offices/europe

[13] Krasniqi, F., Hundozi, B., Pajazitaj, Q. (1981): A contribution to the study of flora of Kosovo. - Bulletin of Faculty of Natural Science Priština VII: 133-139. (Kontribut njohjes së florës së Kosovës, - Bul. i pun. shkenc. i FSHN-Pristinë) (in Albanian).

[14] Krasniqi, E., Millaku, F. (2007): The association Hyperico-Euphorbietum glabriflorae Rexhepi 1978 in the serpentine terrains of Drenica mountain. - Hacquetia 6(2): 183-193.

[15] Krasniqi, E., Berisha, N., Millaku, F., Rexhepi, F. (2019): Contribution to the knowledge on the flora of Mt Golesh, central Kosovo. - Nat. Croat. 28(2): 423-440.

[16] Krivošej, Z., Tatić, B., Atanacković, B., Vasić, P. (1993): Species Rhamnus tinctorius Wald. et Kit. and Aster albanicus Deg. present in the flora of Kosovo and Metohija. $-3^{\text {rd }}$ meeting of the flora of southeaestern Serbia, Proceedings, 1. Flora and Vegetation, Leskovac-Pirot. (Vrste Rhamnus tinctorius Wald. et Kit. i Aster albanicus Deg. prisutne u flori Kosova i Metohije. - III Simpozijum o flori jugoistočne Srbije, zbornik radova 1.flora i vegetacija, Leskovac-Pirot) (in Serbian).

[17] Krivošej, Z., Tatić, B., Gligorijević, S., Atanacković, B. (1995-1998): Floristic increase on the teritory of Kosovo and Metohija (Serbia). - Bulletin of Natural History Museum B49-50: 33-38. (Florističke prinove za teritoriju Kosova i Metohije (Srbija).- Glasnik Prirodnjačkog muzeja u Beogradu, Vol. B 49-50: 33-38) (in Serbian).

[18] Krivošej, Z., Amidžić, L., Lazarević, P., Milinčić, D. (2003): Cheilanthes persica (Bory) Mett. (Polypodiaceae) - new fern species in the flora of Serbia. - Protection of Nature 54(1-2): 17-20. (Cheilanthes persica (Bory) Mett. (Polypodiaceae) nova vrsta paprati u flori Srbije. Zaštita prirode 54/1-2, 17-20) (in Serbian). 
[19] Krivošej, Z., Prodanović, D. (2011): New and rare species of flora of Kosovo and Metohija. - Nature Protection in XXI ${ }^{\text {st }}$ Century, Proceedings of the Conference, Book No 2: $479-484$.

[20] Krivošej, Z., Prodanović, D., Lazarević, P., Vasić, P. (2013): Ophioglossum vulgatum (Ophioglossaceae) in the flora of Kosovo and Metohija (Serbia). - Natura Montenegrina 12(2): 395-404.

[21] Kurt, L., Ozbey, B., Kurt, F., Ozdeniz, E., Bolukbasi, A. (2013): Serpentine Flora of Turkey. - Biological Diversity and Conservation 6(1): 134-152.

[22] Lukić, T., Živković-Bubalo, M., Đerčan, B. (2015): Reliability of internet sources in geography: case study of mountains Studena and Žaračka, Serbia. - J. Geogr. Inst. Cvijic. 65(3): 341-356.

[23] Marin, P. D., Tatić, B. (2001): Serpentine soil and plant diversity, with emphasis balkan Peninsula. - Bocconea 13: 145-150.

[24] Millaku, F., Elezaj, I., Berisha, N. (2018): Sympatric area and ecology of some Tulipa species in the West Balkan Peninsula. - Thaiszia-J. Bot. Košice 28(1): 035-047.

[25] Novák, A. (1926): Ad florae Serbiae cognitionem additamentum primum. - Preslia IV. Praha.

[26] Obratov-Petković, D., Popović, I., Belanović, S., Kadović, R. (2006): Ecobiological study of medicinal plants in some regions of Serbia. - Plant Soil Environ. 52(10): 459-467.

[27] Pavlova, D., Dimitrov, D., Kožuharova, E. (2004): Flora of the serpentine complexes in Eastern Rhodopes (Bulgaria). - Pensoft \& Nat. Mus. Natur. Hist., Sofia, pp. 111-121.

[28] Pavlova, D. K. (2007): Endemics and rare plants growing on serpentines in the Rhodopes Mountains (Bulgaria). - In: Filipovski, G., Lozanovski, R., Matevski, V. (eds.) Collection of papers devoted to academician Kiril Micevski: on the occasion of the 80 years of his birth. Macedonian Acad. Sci. \& Arts, Skopje, Macedonia.

[29] Pavlova, D. K. (2010): A survey of the serpentine flora in the West Bulgarian Frontiers Mts (Mt Vlahina and Mt Ograzhden). - Phytologia Balcanica 16(1): 97-101.

[30] Pavlović, Z. (1967): About one endemic plant association on serpentinite in Ibar river valley. - Bulletin of Institute for Botany and Botanical Garden University of Belgrade II(1-4): 189-195. ( Sur une association végétale endémique des terrains serpentineux dans la vallée de la riviére Ibar (Serbie). - Bulletin de L'Institut et du Jardin Botaniques de L'Université de Beograd) (in French).

[31] Prodanović, D., Amidžić, L., Lazarević, P., Krivošej, Z., Vasić, P. (2004): New localities of the species Fibigia clypeata (L.) Medicus (Brassicaceae) in the flora of Serbia. Protection of Nature 56(1): 53-58. (Novi lokaliteti vrste Fibigia clypeata (L.) Medicus (Brassicaceae) u flori Srbije. - Zaštita prirode 56(1): 53-58.) (in Serbian).

[32] Prodanović, D. (2007): Serpentine flora of Ibarska valley parts in Kosovo. - PhD thesis (manuscript), University of Priština, Kosovska Mitrovica (Serpentinska flora kosovskog dela Ibarske doline,doktorska disertacija (rukopis), Univerzitet u Prištini, Kosovska Mitrovica) (in Serbian).

[33] Prodanović, D., Krivošej, Z., Amidžić, L. (2008): Internationally significant vascular plants in the North of Kosovo and Metohija, the middle course valley of the Ibar river. Natura Montenegrina 7(3): 329-335.

[34] Prodanović, D., Krivošej, Z., Lazarević, P., Amidžić, L. (2010): Contribution to the knowledge of serpentine flora in Kosovo's part of the Ibar river valley. - Botanica Serbica 34(2): 81-86.

[35] Prodanović, D., Krivošej, Z., Amidžić, L. (2012): Ecological features of steppe flora on the Ibar valley serpentine, Northern Kosovo. - Natura Montenegrina 11(3): 405-424.

[36] Prodanović, D., Krivošej, Z., Amidžić, L., Bartula, M. (2013): Floristic and chorological news from northern Kosovo, in the Ibar river valley. - Natura Montenegrina 12(2): 257269. 
[37] Prodanović, D., Krivošej, Z., Stanojević, M., Ćirić, S. (2018): Supplement to the phytogeographical studies of the rare and internationally significant species in the flora of Serbia, Kosovo and Metohija North. - University Thought 8(1): 10-16.

[38] Pustahija, F. (2011): Genome response to abiotic stress: an example of serpentinophytes in central Bosnia. - PhD thesis (manuscript), Sarajevo University, Faculty for Natural Science. (Odgovor genoma na abiotički stres: primjer serpentinofita u centralnoj Bosni. Doktorska disertacija (manuscript). Univerzitet u Sarajevu, Prirodno-matematički fakultet) (in Bosnian).

[39] Rajakaruna, N., Boyd, R. S. (2014): Geoecology. - In: Gibson, D. (ed.) Oxford Bibliographies in Ecology. Oxford University Press, New York.

[40] Ranđelović, N., Rexhepi, F., Jovanović, V. (1982): Contributions to the study of the north-eastern Kosovo flora. - Acta. Bio. Med. Exp. 7: 39-45.

[41] Raunkiaer, C. (1934): The life forms of plants and statistical plant geography. - Claredon press, Oxford.

[42] Rexhepi, F. (1979): Contribution to the serpentinite flora of Kosovo. - Biotehnika 1-2: 53-70. (Kontribut për njohjen e florës së serpentinës në kosovë-in Albanian; Prilog poznavanju flore na serpentinitima Kosova- in Serbian).

[43] Rexhepi, F. (1992): Association Astero-Juniperetum oxycedri Rexhepi 1990. - Bulletin of the Natural History museum in Belgrade B(47): 35-42. (Fitocenoza AsteroJuniperetum oxycedri Rexhepi 1990. - Glasnik prirodnjačkog muzeja u Beogradu Vol B(47): 35-42).

[44] Salihaj, M., Bani, A., Shahu, E., Benizri, E., Echevarria, G. (2018): Metal accumulation by the ultramafic flora of Kosovo. - Ecological Research 33(5): 687-703.

[45] Selvi, F. (2007): Diversity, geographic variation and conservation of the serpentine flora of Tuscany (Italy). - Biodiversity and Conservation 16: 1423-1439.

[46] Shuka, L., Hallaçi, B. (2010): Is determined flora and vegetation of Mirusha (Kosovo) and Kolshi (Albania) area from the serpentine substrate? - Balwois $4^{\text {th }}$ conference -Ohrid, 25-29 May, pp. 1-6.

[47] Sørensen, T. (1948): A method of establishing groups of equal amplitude in plant sociology based on similarity of species and its application of the vegetation on Danish commons. - Biol. Skr. Copenhagen 5(4): 1-3.

[48] Stevanović, V. (1992a): Floristic division of the territory of Serbia with an overview of higher chorion and appropriate floral elements. - In: Sarić, M. R. (ed.) Flora of Serbia 1. Serbian Academy of Science and Art, Belgrade (Floristička podela teritorije Srbije sa pregledom viših horiona i odgovarajućih flornih elemenata. U Flora Srbije 1, Srpska Akademija Nauka i Umetnosti, Beograd) (in Serbian).

[49] Stevanović, V. (1992b): Life form plant classification in Serbian flora. - In: Sarić, M. R. (ed.) Flora of Serbia 1. Serbian Academy of Science and Art, Belgrade (Klasifikacija životnih formi biljaka u flori Srbije. U Flora Srbije 1, Srpska Akademija Nauka i Umetnosti, Beograd) (in Serbian).

[50] Stevanović, V., Jovanović, S., Lakušić, D., Niketić, M. (1995): Diversity of vascular plants of Yugoslavia, with review of internationally significant species. - In: Stevanović, V., Vasić, V. (eds.) Biodiversity of Yugoslavia with an overview of species of global importance. Ecolibri: Faculty of Biology, Belgrade (Diverzitet vaskularne flore Jugoslavije sa pregledom vrsta od međunarodnog značaja.- Biodiverzitet Jugoslavije: sa pregledom vrsta od međunarodnog značaja, Stevanović, V., Vasić, V. (eds.) Ecolibri: Biološki fakultet, Beograd) (in Serbian).

[51] Stevanović, V., Tan, K., Iatrou, G. (2003): Distribution of the endemic Balkan flora on serpentine I.-obligate serpentine endemics. - Plant. Syst. Evol. 242: 149-170.

[52] Stevanović, V., Jakovljević, K. (2014): Serpentinite flora of Serbia. - In: Stevanović, V. et al. (eds.) Josif Pančić-timeless heritage: exibition of 200 of years from borning of great serbian scientist. Galery of science SANU: Faculty of Biology University of Belgrade (Serpentinska flora Srbije. - In Josif Pančić-nasleđe koje ne zastareva: izložba povodom 
200 godina od rođenja velikana srpske nauke. Stevanović V. i dr. (eds.). Galerija nauke i tehnike SANU: Biološki fakultet Univerziteta, Beograd) (in Serbian).

[53] Stojanović, V., Rilak, S., Jelić, I., Perić, R., Saboljević, M., Lazarević, P. (2015): Plants of international importance in the flora of Serbia. - Institute for Nature Conservation of Serbia: Belgrade (Biljke od međunarodnog značaja u flori Srbije. - Zavod za zaštitu prirode Srbije: Beograd) (in Serbian).

[54] Tatić, B. (1969): Flora and vegetation of Mt. Studena planina near Kraljevo. - Journal of Institute of Botany and Botanical Garden, University of Belgrade IV(1-4): 27-72. (Flora i vegetacija Studene planine kod Kraljeva. - Glasnik Botaničkog zavoda i Bašte Univerziteta u Beogradu IV(1-4): 27-72) (in Serbian).

[55] Tatić, B., Krivošej, Z. (1997): Tulipa serbica (Liliaceae), a new species from Serbia. Bocconea 5: 733-736.

[56] The Euro+Med Plantbase (2006-): The information resource for Euro-Mediterranean plant diversity. - Available at: http://ww2. bgbm.org/EuroPlusMed.

[57] The Plant list (2013): Version 1.1. - Published on the Internet at http://theplantlist.org/.

[58] Tumi, F. A. (2013): Bioaccumulation potential of selected plant species of the family Brassicaceae from serpentine habitats in Serbia. - PhD thesis (manusript), University of Belgrade, Faculty of Biology, Belgrade.

[59] Vasić, O., Diklić, N. (2001): The flora and vegetation on serpentinites in Serbia-a rewiew. - Bocconea 13: 151-164.

[60] Westerbergh, A., Saura, A. (1992): The effect of serpentine on the population structure of Silene dioica (Caryophyllaceae). - Evolution 46(5): 1537-1548. 